\title{
A primate specific loss of function polymorphism in TLR2 gene decreases inflammation and protects humans from organ dysfunction in Malaria
}

Aditya K Panda ${ }^{1 \dagger}$, Ratnadeep Mukherjee ${ }^{1 *}$, Bidyut K Das ${ }^{2}$, Rina Tripathy ${ }^{2}$, Ashok K Satapathy ${ }^{3}$, Shobhona Sharma ${ }^{4}$ and Balachandran Ravindran ${ }^{1 *}$

${ }^{1}$ Infectious Disease Biology, Institute of Life Sciences, Bhubaneswar, Odisha, India

${ }^{2}$ Department of Medicine, S.C.B. Medical College, Cuttack, Odisha, India

${ }^{3}$ Regional Medical Research Centre, Bhubaneswar, Odisha, India

${ }^{4}$ Department of Medicine, S.C.B. Medical College, Cuttack, Odisha, India

${ }^{5}$ Tata Institute of Fundamental Research, Mumbai, Maharashtra, India

*Corresponding author:

Balachandran Ravindran, Ph.D.

Infectious Diseases Biology Laboratory,

Institute of Life Sciences, Bhubaneswar- 751023 India;

Phone: +91 674 2304204; Fax: +91 6742300728

E-mail: balaravi@ils.res.in; ravindran8@gmail.com

$\dagger$ Current address: Centre for Life Sciences, Central University of Jharkhand, Brambe, Ranchi, Jharkhand, India

* Current address: National Cancer Institute, NIH, Bethesda, MD, USA

One Sentence Summary: A 23bp deletion in TLR2 gene is associated with high inflammation and susceptibility to organ dysfunction in Plasmodium falciparum malaria. 


\begin{abstract}
Polymorphisms of TLR genes could regulate and contribute functionally to innate immunity and inflammation. TLR2, a promiscous receptor recognizes Pathogen Associated Molecular Patterns from several microbes, bacterial, viral, protozoan and helminths. We demonstrate that monocytes of humans with deletion polymorphism in TLR2 gene (a 23 bp deletion in 5' UTR region) respond more vigorously in vitro to several TLR2 ligands in comparison to those with insertion allele. Lower primates such as Rhesus monkeys and Baboon display 'deletion' genotype while insertion is found in higher primates viz., Orangutan, Chimpanzees and Gorilla. Enhanced inflammation is a hallmark of pathogenesis in human severe malaria leading to bad prognosis and odds ratio of patients prone to develop severe malaira such as multi organ failure with del/del genotype was found to be very high. Based on induction of inflammatory cytokines by normal human PBMCs in vitro and circulating cytokine levels in cohorts of patients with severe $P$. falciparum malaria, we propose that 'insertion' of a 23bp sequence in 5'UTR region of TLR2 gene could have led to moderated TLR2 induced inflammation thus offering survival advantage to higher primates by rendering them relatively refractory to multi-organ dysfunction in severe malaria.
\end{abstract}




\section{Introduction}

Of all the mammalian TLRs characterized so far, TLR2 displays a high degree of promiscuity since PAMPs from several bacterial, viral, fungal, protozoan and helminthic pathogens, and DAMPs such as HMGB1, HSP60, HSP70 etc. stimulate through TLR2 resulting in inflammatory activation of macrophages, the cellular basis of both innate immunity and inflammation $(1,2)$. Polymorphisms in TLR2 gene thus determine clinical outcome of several human diseases (3). A 23 bp insertion/deletion polymorphism in 5' UTR region of TLR2 gene reported in human populations and submitted by us to dbSNP (ss181129011 https://www.ncbi.nlm.nih.gov/projects/SNP/snp_ss.cgi?ss=181129011) was of specific interest to us for two reasons - a) the insertion was located in chromosome 4, approximately $60 \mathrm{bp}$ downstream of an NF-kB binding site, a key region regulating inflammation (4) and b) although published literature describes this polymorphism as Insertion vs Deletion in several ethnic human populations - Ins allele being the dominant one, a search of database revealed that the $23 \mathrm{bp}$ insertion in the 5' UTR region of TLR2 gene was unique and found only in primates and not in rodents and other mammals. More interestingly, in lower primates such as rhesus, nomascus monkeys, baboons etc insertion of one copy of the $23 \mathrm{bp}$ sequence was observed and in higher primates viz., Orangutans and Chimpanzees two copies were found to be inserted while in Gorilla three copies were found (summarized in Figure 1a). In the present study we set out to a) validate these insertion polymorphism in wild primates, and b) test in vitro response of human mononuclear cells with Indel polymorphism on stimulation with several TLR2 ligands, and c) to study the status of inflammatory response in patients with severe malaria to correlate the Indel polymorphism with disease outcome.

\section{Results}

We tested the 23 bp TLR2 polymorphism in 4 rhesus monkeys, 3 baboons, 25 Chimpanzees and 8 Gorilla using fecal DNA samples to validate gene sequences reported in NCBI data-base - polymorphism observed by us was consistent with sequences reported in the database - all the 25 Chimpanzees were homozygous for 'del' allele and 1 of the 8 Gorilla was heterozygous while the other 7 displayed homozygous double 'Insertion' (Figures 1b, 1c and 1d). A closer examination with rodent TLR2 gene sequence revealed that expression of $23 \mathrm{bp}$ 'insertion' and 'deletion' widely being reported by investigators in literature in humans and in 
the data base is a misnomer - the polymorphism is essentially absent in TLR2 5' UTR region of rodent genes and lower primates seems to have acquired one copy since downstream of the $23 \mathrm{bp}$ 'insertion' a similar 23 bp sequence is found in rhesus monkeys, nomascus monkeys and baboon which are otherwise classified as 'deletion' in literature (fig 1a). Thus what has been designated as 'deletion' in lower primates in reality is 'insertion' when compared with 5' UTR region gene of mice TLR2. In higher primates such as Chimpanzee and Organgutan the $23 \mathrm{bp}$ sequence is found duplicated once and in Gorilla TLR2 gene it is duplicated twice. The 'insertion' event acquired by lower primates appears to have been positively selected during evolution. In this manuscript for the sake of clarity with current literature we continue to use the terminology being widely used currently viz., 'deletion' and 'insertion' of 23bp sequence of TLR2 gene.

Since wild life regulations do not allow undertaking phenotypic studies using cells of higher primates, we studied functional significance of this TLR2 polymorphism in humans with 'del' and 'ins' alleles. In a cohort of 432 normal healthy Indian adult population prevalence of 'del' allele was found to be about $14 \%$ - the frequency of 'del' polymorphism in other geographical areas varied from $13 \%$ in PNG to $36 \%$ in Northern Han Chinese. (Table 1). Normal healthy human volunteers with 'del' allele, expressed significantly more surface TLR2 on monocytes in comparison to those with 'ins' allele (Fig 2a) and also displayed elevated plasma TNF- $\alpha$ and CRP levels, (although at physiologically normal levels) markers of systemic inflammation (Fig $2 \mathrm{~b} \& 2 \mathrm{c}$ ). A significant positive correlation was found between plasma levels of TNF- $\alpha$ and CRP (Fig 2d). These findings suggested a positive association between 'del' allele of TLR2 gene and biomarkers of systemic inflammation even in normal healthy adults. We tested the significance of this observation in vitro by directly testing for activation with TLR2 lignads. We stimulated normal human PBMCs with Pam3CSK4 and Vi polysaccharide of Salmonella typhi (5) and quantified cytokines in culture supernatants. Subjects with 'del' allele secreted higher levels of IL-6, IL-1 $\beta$, TNF- $\alpha$ and IL10 in comparison to those homozygous for 'ins' polymorphism (Figure $3 \mathrm{a}, \mathrm{b}, \mathrm{c}, \mathrm{d}$ ). There was no significant difference between the two groups when stimulated with CpG DNA, a TLR9 ligand. For further confirmation a larger panel of TLR2 ligands was used for stimulation of normal PBMCs in vitro. About 4 to 7 fold enhanced secretion of IL-1 $\beta$ was observed in subjects with 'del' allele when stimulated with not only with Pam3CSK4 and Vi polysaccharide but also 
with peptidoglycan (PGN) from 6 different microbial source or $\gamma$-irradiated Mycobaterium tuberculosis (Figure 3e), suggesting propensity of 'del' subjects to respond more vigorously to TLR2 ligands. The observed difference was specific to only TLR2 since TLR4, TLR5 and TLR9 expression were comparable in all the subjects (data not shown) and levels of IL-1b secretion was similar in both the groups when stimulated with poly IC, flagellin or CpG DNA (Figure 2e). We conclude that normal humans with 'del' allele in TLR2 gene display higher levels of plasma TNF- $\alpha$ and CRP and their PBMCs get activated more vigorously when stimulated with TLR2 ligands.

Since the insertion of $23 \mathrm{bp}$ sequence in TLR gene is observed only primates and not in lower animals such as rodents we addressed the issue of significance of this insertion during primate evolution. 'Malaria hypothesis' widely reported in literature attributes severe $P$. falciparum for selection of a variety of haemoglobinopathies reported in human population (6). Secondly, malarial glycolsyl-phosphatidylinositol (GPI), a TLR2 ligand has been proposed as a 'malaria toxin' that induces inflammatory activation of monocytes/macrophages and is reported to mediate systemic inflammation and play a role in mediating severe clinical manifestations observed in malaria $(7,8)$. Based on this logic malarial GPI has also been tested as a candidate anti-disease vaccine for malaria (9). We hypothesized that malaria could have offered survival advantage in patients with insertion allele of $23 \mathrm{bp}$ sequence in their TLR2 gene that leads to decreased inflammatory response thus contributing to lower inflammatory response, lesser clinical severity and mortality caused by malaria. To test our hypothesis we enrolled 398 malaria patients with different grades of clinical severity viz., un-complicated malaria (UM), cerebral malaria (CM), multi organ dysfunction (MOD) and non-cerebral severe malaria (NCSM) (10) (Table 2) and genotyped TLR2 ins/del polymorphism in 356 patients (Table 3). Patients with 'del' polymorphism displayed predilection for development of severe malaria (Table 3) and increased mortality (Table 4). Plasma TNF- $\alpha$ and CRP levels correlated positively with clinical severity (Fig $4 \mathrm{a}$ and $4 \mathrm{~b}$ ) as well as mortality (Fig 4c and 4d) in the cohort of malaria patients analyzed in the study, confirming earlier reports of direct correlation between increased inflammation and bad prognosis in malaria $(11,12)$. More crucially, increased plasma TNF- $\alpha$ and CRP levels in our patients correlated very significantly with TLR2 genotype - patients with 'del' allele were found to be high producers of TNF- $\alpha$ and CRP (Fig $5 \mathrm{a}$ and $5 \mathrm{~b}$ respectively) in comparison to those homozygous for 'ins' polymorphism, 
suggesting a significant and association between this TLR2 polymorphism and enhanced systemic inflammation and clinical severity in human $P$. falciparum malaria. This association was found to be consistent when each of the clinical groups were analyzed for plasma TNF- $\alpha$ (Fig 5c respectively). Polymorphism of TNF- $\alpha$ genes and its association with enhanced TNF- $\alpha$ levels and clinical severity have been reported (13) - in comparison our observations on TLR2 'del' polymorphism in adult population and its association with plasma TNF- $\alpha$ levels as well as clinical severity and mortality appears to be most compelling. Another polymorphism viz., GT repeat microsatellite in intron2 of TLR2 gene has been earlier shown to determine TLR2 mediated inflammation in humans - larger the number of GT repeats lesser is the inflammatory response to TLR2 ligands $(14,15)$. Such GT repeats are absent in lower primates and higher primates display GT repeats (16). We typed our cohort of malaria patients to investigate possible relationship between GT repeat polymorphism and plasma TNF- $\alpha$ and CRP levels. There was no significant association between GT repeat polymorphism and plasma levels of TNF- $\alpha$ and CRP (Fig 6a and 6b respectively). When TLR2 23bp polymorphism and GT repeats were combined and analyzed together, patients with 'del' allele were found to have significantly more TNF- $\alpha$ and CRP in patients regardless of short or long GT repeats in TLR2 gene (Fig 6c and 6d respectively)

\section{Discussion}

The current study has offered several novel insights in the context of a TLR-2 polymorphism, evolution of TLR-2 in primates and susceptibility to TLR2 mediated inflammation in human malaria. First, 'deletion' of a 23bp nucleotide sequence in TLR-2 observed only in lower primates (Rhesus monkey, Baboon, Marmoset and Nomascus) and not in higher primates (Chimpanzees, Gorilla, Orangutan and Neanderthal) as reported in literature appears to be a misnomer - in comparison with TLR2 gene of rodents, the $23 \mathrm{bp}$ sequence was indeed found to be already inserted in 5' UTR region in lower primates that are otherwise designated as 'deletion' in literature. The 'insertion' seems to have been acquired by lower primates and positively selected during evolution that appears to have been duplicated further in higher primates viz., Chimpanzees, Orangutan and Gorilla. Distribution pattern of TLR2 23bp ins/del polymorphism as reported in database, were confirmed by us by typing polymorphism in 4 rhesus monkeys, 3 baboons, 8 Gorillas and 25 Chimpanzees collected from the wild. In the context of very high prevalence of asymptomatic malaria (analogous to asymptomatic 
infections in malaria endemic human population) in wild Chimpanzees and Gorilla (17) and two and three 23bp 'insertion' sequences respectively in Chimpanzee and Gorilla we propose that malaria may have offered evolutionary pressure to select 'Ins' allele of TLR2 gene in primates.

We sought evidence for this proposal by studying a large cohort of malaria patients since 'Malaria hypothesis' widely reported in literature attributes severe $P$. falciparum infections in human communities for selection of a variety of haemoglobinopathies in human population (6). Secondly, malarial glycosylphosphatidylinositol (GPI), a TLR2 ligand has been proposed as a 'malaria toxin' that induces inflammatory activation of monocytes/macrophages and responsible for severe clinical manifestations observed in malaria $(7,8)$. Based on this logic malarial GPI has also been tested as a candidate anti-disease vaccine for malaria (9). We hypothesized that severe malaria could have offered evolutionary pressure needed for selecting 'ins' allele in TLR2 gene in higher primates leading to decreased inflammation and contributing to lower clinical severity and mortality caused by $P$. falciparum malaria. Reports in literature offer credence to this view. Chimpanzees and Gorilla have restricted natural habitat with small population size and about $40-50 \%$ of wild chimpanzees and gorillas have been found infected (analogous to asymptomatic infection status in humans) with $P$. falciparum 'like' malarial parasites $(17,18)$. On the contrary, severe malaria such as cerebral malarial anemia, multi-organ failure etc are common cause of high mortality in human $P$. falciparum malaria (19) mediated by induction of inflammatory cytokine storm particularly high TNF- $\alpha$ levels leading to enhanced clinical severity and mortality $(6,12)$. To test our hypothesis we enrolled 398 malaria patients with grades of clinical severity viz., uncomplicated malaria (UM), cerebral malaria (CM), multi organ dysfunction (MOD) and noncerebral severe malaria (NCSM) (10) and genotyped TLR2 ins/del polymorphism in 398 patients. Patients with 'del' allele displayed increased predilection for development of severe malaria and increased mortality. Inflammatory cytokine storm and high levels of plasma TNF$\alpha$ have been attributed to development of pathology leading to bad prognosis in severe malaria $(6,12)$. In the cohort of malaria patients studied by us, we observed a significant positive association between high TNF- $\alpha$ levels and clinical severity: patients with MOD displayed the highest levels, followed by NCSM and CM. Plasma TNF- $\alpha$ levels were significantly more in patients who succumbed to severe malaria when compared to survivors, corroborating with 
earlier reports $(6,12,20)$. While the role of very high levels of TNF- $\alpha$ in shock and organ failure leading to mortality has been known (21), the role of CRP in P. falciparum malaria is poorly documented in literature. CRP has been shown to bind to infected erythrocytes and clear damaged RBCs from circulation which possibly leads to anemia (22). Further, high levels of CRP has been related with parasite density and malarial severity (23). However the more significant and novel findings of the current study is association between elevated plasma levels of TNF- $\alpha$ and CRP with the TLR2 Indel genotype - patients with 'del' allele were found to be high producers of TNF- $\alpha$ and CRP in comparison to those with 'ins' allele, suggesting that patients with 'deletion' of 23bp sequence of TLR2 gene are prone to enhanced systemic inflammation leading to higher clinical severity when they get infected with $P$. falciparum malaria. Polymorphism of TNF- $\alpha$ genes and its association with enhanced TNF- $\alpha$ levels and clinical severity has been reported (13) - our observations on TLR2 'del' polymorphism in adult population and its association with TNF- $\alpha$ levels as well as clinical severity and mortality appears most compelling. Since we found 'ins' allele consistently in higher primates in the wild (a large population of which harbor malarial parasites in circulation) and association of 'del' allele with increased clinical severity and mortality in humans with P. falciparum, we propose that malaria could have offered evolutionary pressure for selection of the $23 \mathrm{bp}$ sequence in TLR-2 genes in higher primates thus offering them survival advantage.

While analyzing several wild primates we found consistent presence of 'del' allele in all lower primates and absence of 'del' allele in all Chimpanzees and Gorilla. It is not clear to us currently how about $20 \%$ of humans display 'del' allele in several ethnic human populations. The results do not however rule out complete absence of 'del' allele in higher primates since we have tested only a limited number of Chimpanzees and Gorilla that had survived selection under wild forest conditions. The other possibility is that presence of 'del' allele in about $20 \%$ of humans suggests that selection due to severe forms of malaria may have diminished in early humans due to absence of severe malaria leading to loss of 'ins' allele since it has been argued that emergence of severe $P$. falciparum malaria is a more recent evolutionary event $(24,25)$. It has been proposed that early homo sapiens lost their ability to synthesize N-Glucoryl neuraminic acid and consequently became resistant to $P$. reichnowi (malarial parasites infecting Chimpanzees) until emergence of $P$. falciparum, that uses $\mathrm{N}$ Acetyl neuraminic acid as its receptor (25). Based on genetic analysis of several strains of $P$. 
falciparum, mosquito biting behavior and emergence of haemoglobin disorders selected by $P$. falciparum in Africa it has also been suggested that virulent $P$. falciparum may have emerged in human population only about 5000-10000 years ago as a consequence of introduction of agrarian practice $(24)$.

The possibility that PBMCs with 'del' allele are prone to enhanced activation by TLR2 ligands was also tested. Stimulation of PBMCs of normal healthy volunteers with TLR-2 ligands such as PAM3CSK4, peptidoglycans from several bacterial species, Vi polysaccharide of Salmonella typhi etc revealed that subjects with 'del' allele respond more vigorously releasing significantly high levels of IL-1 $\beta$, IL-6 and TNF- $\alpha$ when compared to those with 'ins' allele. These findings are in contrast to two earlier reports using experimentally transfected cell lines and scoring luciferase activity $(26,27)$ - we attribute this to possible experimental artifacts while using cell line models for studying functional activation of immune cells. Similar discrepancy between transfection-based functional analysis and in vivo response has been reported for TIRAP S180L polymorphism (28). We conclude that transfection-based experiments need to be interpreted with caution while correlating genotypes with phenotypes.

Another polymorphism in intron 2 region of TLR2 gene GT repeat microsatellite has been shown to determine TLR2 mediated inflammation in humans - larger the number of GT repeats lesser is the inflammatory response to TLR2 ligands (15). In the present study, no significant association of TLR2 GT repeats with plasma TNF- $\alpha$ and CRP was observed. Combined analysis of 'del' allele and GT repeat of TLR2 revealed that 'del' allele plays a more important role in induction of increased TNF- $\alpha$ and CRP levels in malaria than GT repeat polymorphism. More significantly, combined distribution of $23 \mathrm{bp}$ ins/del and GT repeat polymorphism reveled that larger GT repeats (reported to be less inflammatory) are linked to 'ins' allele. Our observation of absence of such GT repeats in lower primates and presence only in higher primates, while corroborating with a previous report (16) also supports the notion that decreased TLR2 mediated inflammation is linked to primate evolution. Regardless of malaria contributing to positive selection and emergence of 'ins' allele of TLR2 gene in higher primates, the observation that humans with 'del' allele are prone to increased TLR2 mediated inflammation has opened up avenues for future investigations in human innate immunity/inflammation. Both innate as well as adaptive immune responses are directly influenced by TLR-2 mediated activation of dendritic cells and T lymphocytes (29-31) and 
thus 'ins' vs 'del' in TLR2 gene could influence host response and susceptibility of humans to several other diseases such as HIV-AIDS (32), H. pylori mediated gastric cancer (33) and Alzheimer's disease (34).

\section{Materials and Methods}

\section{Ethics statement}

Human blood samples from healthy controls (laboratory volunteers) and $P$. falciparum infected patients were collected using standardized Institutional protocol approved by Human ethical committee of Institute of Life Sciences, Bhubaneswar. Written informed consent was obtained from each of study participants or accompanying persons in the case of children and patients in comatose state.

\section{Healthy controls}

123 healthy laboratory volunteers from Institute of Life Sciences and Regional Medical Research Centre, Bhubaneswar, India were enrolled. About 50ul of blood was collected by finger prick and genomic DNA was purified using a kit according to manufacturers' instructions (Sigma). All individuals were genotyped for TLR2 (23bp ins/del), TLR2 codon (Arg677Trp, Arg753Gln), TLR4 (Asp299Gly, Thr399Ile) and TLR1 (I602S) polymorphisms as described below. To avoid confounders of activation by TLR2 ligands only those healthy subjects with wild type TLR4 and TLR1 polymorphism were used to investigate phenotype differences between 23bp Indel polymorphism. In addition, 309 healthy controls were enrolled and genotyped for TLR2 indel polymorphism.

\section{Detection of TLR2 (23bp ins/del, Arg753Gln and Arg677Trp) polymorphisms}

All subjects were genotyped for TLR2 (23bp ins/del) polymorphism by polymerase chain reaction as described earlier (35). In brief, following primers were used for (23bp ins/del) genotyping: sense 5'-CACGGAGGCAGCGAGAAA-3' and anti-sense 5'CTGGGCCGTGCAAAGAAG-3'. PCR was carried out in a total reaction volume of $20 \mu \mathrm{L}$ containing about $200 \mathrm{ng}$ genomic DNA, 10 pmol each primer, $200 \mathrm{ng}$ each dNTP and $1 \mathrm{U}$ Taq DNA polymerase (sigma). The thermo-cycling condition was as follows: initial denaturation for $5 \mathrm{~min}$ at $95^{\circ} \mathrm{C}$, followed by 35 cycles of $95^{\circ} \mathrm{C}$ for $30 \mathrm{~s}, 60^{\circ} \mathrm{C}$ for $30 \mathrm{~s}$, and $72^{\circ} \mathrm{C}$ for $30 \mathrm{~s}$. The final extension step was prolonged to $7 \mathrm{~min}$ at $72^{\circ} \mathrm{C}$. PCR products were separated on $3 \%$ 
agarose gel electrophoresis and visualized in gel documentation system after ethidium bromide staining. A single band at $286 \mathrm{bp}$ was classified as wild type (Ins/Ins), a single 263-bp band as homozygous mutation (Del/Del), and those displaying two bands of 286bp and 263bp were as heterozygous mutation (Ins/Del). Randomly chosen PCR products were purified using Qiagen PCR Purification System (Qiagen, UK) and sequenced using an ABI Prism 310 Genetic Analyzer (Applied Biosystems, Foster City, CA) and Big Dye Terminator DNA sequencing kit (Applied Biosystems). Distributions of two TLR2 polymorphisms (Arg753Gln, Arg677Trp) samples were genotyped by PCR followed by restriction fragment length polymorphism (RFLP) according to an earlier report (36). Primers: Forward 5'GCCTACTGGGTGGAGAACCT-3' and Reverse; 5'-GGCCACTCCAGGTAGGTCTT-3'were used to amplify a region of 340 bp including both polymorphisms (Arg753Gln, Arg677Trp) by following conditions: $940 \mathrm{C}$ for $5 \mathrm{~min}$, then 30 cycles of $940 \mathrm{C}$ for $30 \mathrm{sec}, 580 \mathrm{C}$ for $30 \mathrm{sec}$, and $72^{0} \mathrm{C}$ for $30 \mathrm{sec}$. The amplicons were digested overnight with AciI and analyzed by agarose gel electrophoresis. Amplified products were randomly sequenced for confirmation of individual's genotype.

\section{Determination of TLR2, TLR4, TLR5 and TLR9 expression on circulating human} monocytes

The TLR2 expression on cell surface was determined by flow cytometry using two-color immunofluorescent technique in 48 healthy laboratory volunteers: 27 ins/ins individuals, 16 subjects of ins/del and 5 subjects of del/del type were included for analysis. Briefly, 50ul of whole blood were directly stained for $30 \mathrm{~min}$ at $4{ }^{\circ} \mathrm{C}$ with a combination of fluorochromeconjugated mAbs: phycoerythrin (PE)-labeled anti-TLR2 (Imgenix), fluoresceinisothiocyanate (FITC)-labeled anti-CD14 (eBiosciences). Appropriate isotype controls (eBiosciences) were also used. Erythrocytes were lysed using FACS lysis solution (BD Biosciences, USA) and the cells were washed with washing buffer followed by centrifugation at $300 \times \mathrm{g}$ for $5 \mathrm{~min}$. The cell pellet was then re-suspended in $500 \mu \mathrm{l}$ of sheath fluid and analysed flow cytometry in FACS caliber. Expression of TLR4, TLR5 and TLR9 on monocytes was quantified in 10 subjects with ins/ins and 10 with ins/del. About 50ul of whole blood was stained with anti-CD14-FITC and anti-TLR4-PE (eBiosciences) or TLR5-PE (Santacruz), expression was analysed as described above by FACS. For TLR9 staining, cells 
were labeled with anti-TLR9-FITC (eBiosciences) and a membrane marker anti-CD14-PE (eBiosciences), fixed and permeabilized, and analysed by flow cytometry.

\section{Genotyping of TLR4 (Asp299Gly, Thr399Ile) and TLR1 (I602S) polymorphisms}

Genomic DNA of all healthy lab volunteers were analyzed for TLR4 (Asp299Gly, Thr399Ile) and TLR1 (I620S) polymorphisms. Genotyping of TLR4 Asp299Gly polymorphism was carried out by allele specific PCR. Two forward primer (allele specific discrimination at the 3' end) 5'-CTTAGACTACTACCTCGATGA-3' (wild), and 'CTTAGACTACTACCTCGATGG-3' (mutant), with a common anti-sense Reverse Primer5'-TAAGCCTTTTGAGAGATTTGA-3' were used and PCR was performed as follows: 12 cycles of 10 seconds at $950 \mathrm{C}$ followed by 60 seconds at $650 \mathrm{C}$, followed by 17 cycle of 10 seconds at 950C, 50 seconds at 600C and 30 seconds at $720 \mathrm{C}$. The final extension was for 7 min at $720 \mathrm{C}$. $\beta$-actin primers were taken as internal controls. The amplified products (192bp) were analyzed by electrophoresis on $2.5 \%$ agarose gels stained with ethidium bromide. DNA from normal subjects will get amplified with 'wild type' primers - heterozygous mutations will be characterized by amplification with both 'wild type' as well as 'mutation' specific primers. DNA from individuals homozygous for TLR4 mutations will get amplified only with mutant specific primer. TLR4 Thr399Ile polymorphism was detected by PCR amplification of involved region with sense primer 5'-GCTGTTTTCAAAGTGATTTTGGGAGAA-3' that contains a mismatch at position -3 from the 3 , end of the primer ( $G$ instead of $C$ ) to create a HinfI cleavage site when the mutant allele is amplified. Antisense primer was 5'CACTCATTTGTTTCAAATTGGAATG-3'. The parameters were an initial denaturation at $95^{\circ} \mathrm{C}$ for $7 \mathrm{~min}$, followed by 35 cycles: denaturation at $95^{\circ} \mathrm{C}$ for $30 \mathrm{~s}$, annealing at $62^{\circ} \mathrm{C}$ for 30 $\mathrm{s}$, and elongation at $72^{\circ} \mathrm{C}$ for $32 \mathrm{~s}$. The final elongation was at $72^{\circ} \mathrm{C}$ for $5 \mathrm{~min}$ followed for a cooling to $4^{\circ} \mathrm{C}$. The amplicon of 147 -bp length were digested at $37^{\circ} \mathrm{C}$ with Hinf I for 4 hrs resulting in fragments that either were cut into two fragments of 122-bp and 25-bp (mutant allele) or were not restricted (wild allele). These fragments were analyzed by electrophoresis on $3 \%$ agarose gels stained with ethidium bromide. PCR-RFLP technique was also employed to determine variation in codon 602 of TLR1 gene. A fragment containing exon 4 of TLR1 was amplified from genomic DNA using the following PCR primers: forward, CTTGATCTTCACAGCAATAAAATAAAGAGCATTCC, and reverse, 
GGCCATGATACACTAGAACACACATCACT. The PCR conditions were an initial denaturation at $95^{\circ} \mathrm{C}$ for $5 \mathrm{~min}$, followed by 32 cycles: denaturation at $95^{\circ} \mathrm{C}$ for $30 \mathrm{~s}$, annealing at $60^{\circ} \mathrm{C}$ for $30 \mathrm{~s}$, and elongation at $72^{\circ} \mathrm{C}$ for $32 \mathrm{~s}$. The final elongation was at $72^{\circ} \mathrm{C}$ for $5 \mathrm{~min}$. The amplicon was digested by PstI to discriminate between the TLR1 602 alleles.

\section{TLR2 GT repeats typing}

To amplify a region of about 150 base pairs flanking the microsatellite GT repeat, We performed PCR using the FAM-labeled primer (forword-FAM-5' TATCCCCATTCATTCGTTCCATT-3') and a reverse primer (5'GACCCCCAAGACCCACACC-3') (Integrated DNA technologies, Iowa USA). The thermal cycler conditions were as follows: preheating at $94^{\circ} \mathrm{C}$ for 5 minutes, followed by 35 cycles of $94^{\circ} \mathrm{C}$ for 30 seconds, $58^{\circ} \mathrm{C}$ for 30 seconds, and $72^{\circ} \mathrm{C}$ for 30 seconds; and then a final extension of 5 minutes at $72^{\circ} \mathrm{C}$. The numbers of GT repeats were determined by sizing the PCR products using an ABI 310 sequencer (Applied Biosystems, Foster City, CA) and Genescan analysis 2.1 software (Applied Biosystems). For all primates, same primer set were used for typing of TLR2 microsatellite GT repeats.

\section{Isolation and stimulation of monocyte}

About $5 \mathrm{ml}$ of whole venous blood were collected from pre-genotyped healthy lab volunteers that include: seven ins/ins individuals, seven subjects of ins/del genotype and five homozygous del/del individuals. All subjects enrolled for functional study with ins/ins, ins/del or del/del genotypes were wild type for TLR4 (Asp299Gly and Thr399Ile) and TLR1 (I602S) polymorphisms. $5 \mathrm{ml}$ of blood was layered onto $5 \mathrm{ml}$ of HISTOPAQUE-1077 (Sigma) and centrifuged at $400 \mathrm{~g}$ for $30 \mathrm{~min}$ in room temperature. Peripheral blood mononuclear cells were removed from the interface and washed twice (10 $\mathrm{min}, 250 \mathrm{~g}$ ) in culture medium before being counted. Cells were resuspended to $10^{6}$ cells per $\mathrm{ml}$ in Dulbecco's Modified Eagle Medium (DMEM) with 1\% antibiotics (Penicillin Streptomycin solution from Sigma) and 10\% fetal bovine serum. Cells were placed in a 96 well culture plate $(100 \mu 1$ per well) and incubated in a $5 \% \mathrm{CO}_{2}$ incubator at $37^{\circ} \mathrm{C}$ for 4 hours. Non adherent cells were removed by rinsing of medium thrice. Adherent cells were stimulated with wide range of TLR2 agonists: Peptidoglycan from Streptomyces species, Methanobacterium species, Saccharomyces cerevisiae, Micrococcus luteus, Bacillus subtilis, and Staphylococcus aureus $(100 \mu \mathrm{g} / \mathrm{ml})$, all obtained from Sigma. 


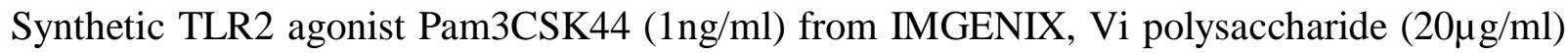
from GlaxoSmithKline and $\gamma$ - irradiated Mycobacterium tuberculosis $\left(1 \mathrm{X} 10^{6} / \mathrm{ml}\right)$. LPS was taken as positive control $(10 \mu \mathrm{g} / \mathrm{ml})$ (Sigma) and monocytes were stimulated with $\mathrm{CpG}$ $(10 \mu \mathrm{g} / \mathrm{ml})$, Poly IC $(10 \mu \mathrm{g} / \mathrm{ml})$ and flagellin $(100 \mu \mathrm{g} / \mathrm{ml})$ as negative control. LPS contamination was minimized by addition of $10 \mu \mathrm{g} / \mathrm{ml}$ of polymixin-B to the culture medium except positive control well (LPS 10ug/ml Sigma). After 48 hours of incubation supernatant were collected and stored at $\quad-80^{\circ} \mathrm{C}$ until assays for cytokine.

\section{Primates DNA}

Blood samples of four rhesus monkeys and three baboons (National institute of Immunology, New Delhi) were collected and whole genomic DNA was isolated by DNA isolation kit (Sigma). Fecal samples of five chimpanzees were collected from captivated animals at Nandan Kanan Zoo, Bhubaneswar, Odisha, India. Fecal DNA was extracted by commercial kit according to manufracturer's instructions (Qiagen). Fecal DNA of 25 Chimpanzees and 8 Gorilla were a kind gift from Prof. B.Hann.

\section{Multiple sequence alignment}

Nucleotide sequence flanking 23bp ins/del polymorphic site of TLR2 gene of all primates whose whole genome sequence data are available in NCBI or Ensembl data base were obtained (Homo sapiens, Pan troglodytes, Pongo abelii, Macaca mulatta, Callithrix jacchus, Papio hamadryas). Recently, Neanderthal genome sequence was reported by sequencing of three Neanderthal bones from the Vindija Cave in Croatia: Vi33.16, Vi33.25, and Vi33.26. The whole genome sequence consists of approximately 50bp short sequence fragments. These sequences were downloaded from database. Our sequencing data (del/del genotype of Homo sapiens, eight sequence of Gorilla gorilla, four Macaca mulatta, three Papio hamadryas and five Pan troglodytes) were aligned using the ClustalW program. All default parameters of ClustalW program were used.

\section{P. falciparum malaria patients}

P. falciparum infected subjects were enrolled at S.C.B. Medical College and Hospital, Cuttack, Odisha. The area is highly endemic for malaria with more than $85 \%$ of malaria cases attributed to $P$. falciparum. Patients enrolled in the current study are residents of coastal districts in India which are highly endemic for P. falciparum malaria - average Annual parasite index (API) of $P$ falciparum was 6.67 (http://nvbdcp.gov.in/Doc/malaria_situation_april12.pdf). Clinically 
suspected malaria patients were screened for P. falciparum infection by Giemsa-stained thick and thin blood smears and immune chromatography test (SD Bio Standard Diagnostics India). Detection of $P$. falciparum was also performed by nested polymerase chain reaction (PCR) (10). P. falciparum infected individuals were categorized based on WHO guidelines. The inclusion criteria for uncomplicated malaria (UM) cases were symptoms of fever with evidence of $P$. falciparum infection in the blood. Severe malaria (SM) patients were categorized into three groups based on distinct clinical features: 1) Cerebral malaria (CM), 2) Non cerebral severe malaria (NCSM) and 3) Multi-organ-dysfuction (MOD) as described in our earlier reports $(10,37)$. About $5 \mathrm{ml}$ of venous blood was collected in EDTA vials from all enrolled patients. Plasma was separated a stored at $-80^{\circ} \mathrm{C}$ until further use.

\section{C-reactive protein (CRP) and Cytokines (TNF- $\alpha, I L 1 \beta)$ assays}

Plasma CRP of healthy lab controls and P. falciparum infected patients were quantified by quantitative immunoturbidometric assays according to manufacturer's instructions (Labkit, Merck, Millipore). Enzyme linked immunosorbent assay (ELISA) was employed for quantification of plasma TNF- $\alpha$ from healthy lab controls and falciparum infected patients according to manufracturer instructions (ebiosciences). IL1 $\beta$ levels in culture supernatant were also quantified by bioplex (Biorad) or ELISA kit (ebiosciences).

\section{Statistical analysis}

GraphPad Prism (Version 5.01) was used for all statistical analysis in the study. Clinical characteristics and biochemical analysis of patients were compared by one-way analysis of variance (ANOVA). All cytokines and plasma CRP data were analyzed using student's t test. Correlation between plasma TNF- $\alpha$ and CRP was analyzed by Spearman's rank test. Allele and genotype frequencies were calculated by direct counting. Fisher's exact test was used to analyze distribution of allele and genotype frequencies with in different groups of $P$. falciparum malaria infected patients and association with mortality. In addition, odds ratios (ORs) and $95 \%$ confidence intervals (CIs) were calculated. Deviations from the HardyWeinberg equilibrium (HWE) were tested using the web-based site http://www.oege.org/software/hwe-mr-calc.shtml. Statistical analysis of P value less than 0.05 was considered as significant. 


\section{References and Notes:}

1. T. Kawai, S. Akira, The role of pattern-recognition receptors in innate immunity: update on Toll-like receptors. Nature immunology 11, 373-384 (2010).

2. C. Ospelt, S. Gay, TLRs and chronic inflammation. The international journal of biochemistry \& cell biology 42, 495-505 (2010).

3. N. W. Schroder, R. R. Schumann, Single nucleotide polymorphisms of Toll-like receptors and susceptibility to infectious disease. The Lancet. Infectious diseases 5, 156-164 (2005).

4. C. M. Johnson, R. I. Tapping, Microbial products stimulate human Toll-like receptor 2 expression through histone modification surrounding a proximal NF-kappaB-binding site. The Journal of biological chemistry 282, 31197-31205 (2007).

5. R. Garg, A. Qadri, Hemoglobin transforms anti-inflammatory Salmonella typhi virulence polysaccharide into a TLR-2 agonist. Journal of immunology 184, 5980-5987 (2010).

6. T. E. Wellems, K. Hayton, R. M. Fairhurst, The impact of malaria parasitism: from corpuscles to communities. The Journal of clinical investigation 119, 2496-2505 (2009).

7. D. C. Gowda, TLR-mediated cell signaling by malaria GPIs. Trends in parasitology 23, 596-604 (2007).

8. F. Kamena et al., Synthetic GPI array to study antitoxic malaria response. Nature chemical biology 4, 238240 (2008).

9. L. Schofield, M. C. Hewitt, K. Evans, M. A. Siomos, P. H. Seeberger, Synthetic GPI as a candidate antitoxic vaccine in a model of malaria. Nature 418, 785-789 (2002).

10. A. K. Panda et al., Association of ABO blood group with severe falciparum malaria in adults: case control study and meta-analysis. Malaria journal 10, 309 (2011).

11. I. A. Clark, A. C. Budd, L. M. Alleva, W. B. Cowden, Human malarial disease: a consequence of inflammatory cytokine release. Malaria journal 5, 85 (2006).

12. D. Kwiatkowski et al., TNF concentration in fatal cerebral, non-fatal cerebral, and uncomplicated Plasmodium falciparum malaria. Lancet 336, 1201-1204 (1990).

13. W. McGuire, A. V. Hill, C. E. Allsopp, B. M. Greenwood, D. Kwiatkowski, Variation in the TNF-alpha promoter region associated with susceptibility to cerebral malaria. Nature 371, 508-510 (1994).

14. N. C. Suryadevara et al., Influence of Intron II microsatellite polymorphism in human toll-like receptor 2 gene in leprosy. Human immunology 74, 1034-1040 (2013).

15. M. Veltkamp et al., Linkage between Toll-like receptor (TLR) 2 promotor and intron polymorphisms: functional effects and relevance to sarcoidosis. Clin Exp Immunol 149, 453-462 (2007).

16. J. J. Yim, A. A. Adams, J. H. Kim, S. M. Holland, Evolution of an intronic microsatellite polymorphism in Toll-like receptor 2 among primates. Immunogenetics 58, 740-745 (2006).

17. W. Liu et al., Origin of the human malaria parasite Plasmodium falciparum in gorillas. Nature 467, 420425 (2010).

18. F. Prugnolle et al., African great apes are natural hosts of multiple related malaria species, including Plasmodium falciparum. Proceedings of the National Academy of Sciences of the United States of America 107, 1458-1463 (2010).

19. J. Langhorne, F. M. Ndungu, A. M. Sponaas, K. Marsh, Immunity to malaria: more questions than answers. Nat Immunol 9, 725-732 (2008).

20. R. Manish, R. Tripathy, B. K. Das, Plasma glucose and tumour necrosis factor-alpha in adult patients with severe falciparum malaria. Trop Med Int Health 8, 125-128 (2003).

21. D. L. Stevens, Group A streptococcus--from basic science to clinical disease. West J Med 164, 25-27 (1996).

22. W. Ansar, S. M. Bandyopadhyay, S. Chowdhury, S. H. Habib, C. Mandal, Role of C-reactive protein in complement-mediated hemolysis in Malaria. Glycoconjugate journal 23, 233-240 (2006).

23. S. H. Gillespie et al., Measurement of acute phase proteins for assessing severity of Plasmodium falciparum malaria. Journal of clinical pathology 44, 228-231 (1991).

24. R. Carter, K. N. Mendis, Evolutionary and historical aspects of the burden of malaria. Clinical microbiology reviews 15, 564-594 (2002).

25. N. M. Varki, E. Strobert, E. J. Dick, Jr., K. Benirschke, A. Varki, Biomedical differences between human and nonhuman hominids: potential roles for uniquely human aspects of sialic acid biology. Annual review of pathology 6, 365-393 (2011). 
26. E. Noguchi et al., An association study of asthma and total serum immunoglobin E levels for Toll-like receptor polymorphisms in a Japanese population. Clin Exp Allergy 34, 177-183 (2004).

27. A. Junpee, T. Tencomnao, V. Sanprasert, S. Nuchprayoon, Association between Toll-like receptor 2 (TLR2) polymorphisms and asymptomatic bancroftian filariasis. Parasitol Res 107, 807-816 (2010).

28. B. Ferwerda et al., Functional and genetic evidence that the Mal/TIRAP allele variant $180 \mathrm{~L}$ has been selected by providing protection against septic shock. Proc Natl Acad Sci U S A 106, 10272-10277 (2009).

29. S. Manicassamy et al., Toll-like receptor 2-dependent induction of vitamin A-metabolizing enzymes in dendritic cells promotes T regulatory responses and inhibits autoimmunity. Nature medicine 15, 401-409 (2009).

30. T. Imanishi et al., Cutting edge: TLR2 directly triggers Th1 effector functions. Journal of immunology 178, 6715-6719 (2007).

31. J. M. Reynolds et al., Toll-like receptor 2 signaling in CD4(+) T lymphocytes promotes T helper 17 responses and regulates the pathogenesis of autoimmune disease. Immunity 32, 692-702 (2010).

32. S. Vidyant, A. Chatterjee, V. Agarwal, T. N. Dhole, Susceptibility to HIV-1 infection is influenced by toll like receptor-2 (-196 to -174) polymorphism in a north Indian population. The journal of gene medicine 19, (2017).

33. N. Castano-Rodriguez, N. O. Kaakoush, K. L. Goh, K. M. Fock, H. M. Mitchell, The role of TLR2, TLR4 and CD14 genetic polymorphisms in gastric carcinogenesis: a case-control study and meta-analysis. PloS one 8, e60327 (2013).

34. J. T. Yu, S. M. Mou, L. Z. Wang, C. X. Mao, L. Tan, Toll-like receptor $2-196$ to -174 del polymorphism influences the susceptibility of Han Chinese people to Alzheimer's disease. Journal of neuroinflammation 8, 136 (2011).

35. T. Tahara et al., Toll-like receptor 2 -196 to $174 \mathrm{del}$ polymorphism influences the susceptibility of Japanese people to gastric cancer. Cancer science 98, 1790-1794 (2007).

36. A. Berdeli, H. A. Celik, R. Ozyurek, B. Dogrusoz, H. H. Aydin, TLR-2 gene Arg753Gln polymorphism is strongly associated with acute rheumatic fever in children. Journal of molecular medicine 83, 535-541 (2005).

37. A. K. Panda et al., Complement receptor 1 variants confer protection from severe malaria in Odisha, India. PloS one 7, e49420 (2012).

\section{Acknowledgments:}

The authors thank Dr. Krishna Prasad, AIIMS, New Delhi for generous gift of $\gamma$-irradiated M. tuberculosis, Dr. Subeer Majumdar, NII, Delhi for samples of normal Rhesus monkeys, Dr. D. K. Panda, Director, Nandankanan zoo, Bhubaneswar for stool samples of Chimpanzees, Prof. Beatrice Hahn, University of Pennsylvania, USA for generous gift of DNA samples of Gorilla and Chimpanzees and Dr. Tushar Vaidya, CCMB, Hyderabad for assisting in analysis of Neanderthal sequences and Prof. Partha Majumder for critically reading the manuscript.

Funding: Institute of Life Sciences, Department of Biotechnology, Government of India

\section{Author contributions:}

Competing interests: The authors declare no conflict of interest. 
bioRxiv preprint doi: https://doi.org/10.1101/239129; this version posted January 18,2018 . The copyright holder for this preprint (which was not certified by peer review) is the author/funder, who has granted bioRxiv a license to display the preprint in perpetuity. It is made available under aCC-BY-NC-ND 4.0 International license.

\begin{abstract}
Figures
a:

Human (ins)

Human (del)

Chimpanzee $\mathrm{db}$

Orangutan $\mathrm{db}$

Marmoset $d b$

Nomascus $d b$

Rhesus $\mathrm{db}$

Baboon $\mathrm{db}$

Neanderthal (Vi33.25)

GAAAGCGCAGCCAGGCGGCTGCTCGGCGTTCTCTCAGGTGACTGCTCGGAGTTCTCCCAGGTACGT GAAAGCGCAGCCAGG - - - - - GAAAGCGCAGCCAGGCGGCTGCTCGGCGTTCTCTCAGGCAACTGCTCGGCGTTCTCCCAGGTACGT GAAAGCGCAGACAGGCGGCTGTTCGGCGTTCTCCCAGGCGGCTGTTCGGCGTTCTCCCAGGTACGT

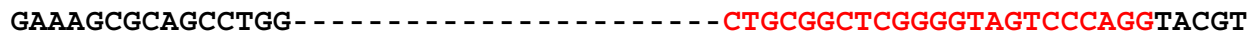
GAGAGCTCAGCCAGG- - GAAAGCTCAGCCAGG - - - - - CGGCTGCTCCGCGTCCTCCCAGGTAAGT Neanderthal (Vi33.26) Gorilla db GAAAGCGCAGCCAGGCGGCTGCTCGGCGTTCTCTCAGGTGGCTGCTCGGCGTTCTCCCAGGCGGCTGCTCGGCGTTCTCCCAG GTACGT

Mouse
\end{abstract}

b:

rhesus 1

rhesus 2

rhesus 3

rhesus 4

baboon 1

baboon 2

baboon 3
GAAAGCTCAGCCAGG---GAAAGCTCAGCCAGG------n GAAAGCTCAGCCAGG------n GAAAGCTCAGCCAGG---n GAAAGCTCAGCCAGG--n

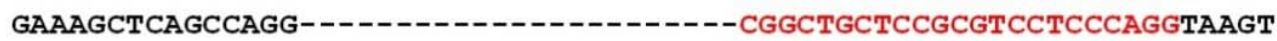

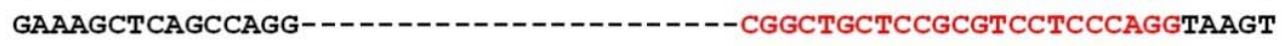


c:

Nandankanan C1
Nandankanan C2
Nandankanan C3
Nandankanan C4
Nandankanan C5
Chimp GT320
Chimp GT401
Chimp GT605
Chimp GT606
Chimp GT607
Ch1mp GT616
Chimp GM2544
Ch1mp GM2563
Chimp GM2586
Ch1mp GL2634
Chimp GM2690
Ch1mp GM2691
Chimp BA2872
Chimp BE0983
Chimp EP0927
Chimp KA2261
Chimp LU1941
Chimp PA0340
Chimp UB2502
Chimp WA0351

GAAAGCGCAGCCAGGCGGCTGCTCGGCGTTCTCTCA GGCA GAAAGCGCAGCCAGGCGGCTGCTCGECGTTCTCTCAGGCAACTECTCGGCGITCTCCCAGGTACGT GAAAGCGCAGCCAGGCGGCTGCTCGGCGTTCTCTCAGGCAACTGCTCGGCGTTCTCCCAGGTACGT GAAAGCGCAGCCAGGCEGCTGCTCGECGTTCTCTCAGGCAACTECTCGGCGTTCTCCCAGGTACGT GAAAGCGCAGCCAGGCGGCTGCTCGGCGTTCTCTCAGGCAACTGCTCGGCGTTCTCCCAGGTACGT GAAAGCGCAGCCAGGCGGCTGCTCGECGTTCTCTCAGGCAACTECTCGGCGTTCTCCCAGGTACGT GAAAGCGCAGCCAGGCGGCTGCTCGGCGTTCTCTCAGGCAACTGCTCGGCGTTCTCCCAGGTACGT GAAACCCAGCCAGGCGGCTECTCGGCGITCTCTCAGGCAACTECTCGGCGTICTCCCAGGTACGT GAAAGCGCAGCCAGGCGGCTGCTCGGCGTTCTCTCAGGCAACTGCTCGGCGTTCTCCCAGGTACGT GAAAGCGCAGCCAGGCGGCTGCTCGGCGTTCTCTCAGGCAACTGCTCGGCGTTCTCCCAGGTACGT GAAAGCGCA GCCAGGCGGCTGCTCGGCGTTCTCTCAGGGAACTGCTCGGCGTTCTCCCAGGTACGT GAAAGCGCAGCCAGGCGGCTGCTCGGCGTTCTCTCAGGCA ACTGCTCGGCGTTCTCCCAGGTACGT GAAAGCGCAGCCAGGCGGCTGCTCGGCGTTCTCTCAGGCAACTGCTCGGCGTTCTCCCAGGTACGT GAAAGCGCAGA CAGGCGGCTGCTCGGCGTTCTCTCAGGCA TCTGCTCGGCGTTCTCCCAGGTACGT GAAAGCGCAGCCAGGCGGCTECTCGGCGTTCTCTCA GGCAACTGCTCGGCGTTCTCCCAGGTACGT GAAAGCGCAGCCAGGCGGCTGCTCGGCGTTCTCTCAGGCA CTECTCGGCGTTCTCCCAGGTAGGT GAAAGCGCAGCCAGGCGGCTECTCGEC GTTCTCTCAGGCAACTECTCGGCGTTCTCCCAGGTACGT GAAAGCGCAGCCAGGCGGCTGCTCGGCGTTCTCTCAGGCARCTGCTCGGCGTTCTCCCAGGTACGT GAAAGCGCAGCCAGGCGGCTGCTCGSCGTTCTCTCAGGCAATTCTCGGCGTTCTCCCAGGTACGT GAAAGCGCAGCCAGGCGGCTGCTCGGCGTTCTCTCAGGCAACTGCTCGGCGTCTCCCAGGTAAGT GAAAGCGCAGCCAGGCGGCTGCTCGECGTTCTCTCAGGCAACTGCTCGGCGTICTCCCAGGTACGT GAAAGCGCAGCCAGGCGGCTGCTCGGCGTTCTCTCAGGCAACTGCTCGGCGTCTCCCAGGTACGT GAAAGCGTAGCCAGGCGGCTGCTCGECGTTCTCTCAGGCAACTGCTCGGCGTTCTCCCAGGTACGT GAAAGCGCAGCCAGGCGGCTGCTCGGCGTTCTCTCAGGCAACTGCTCGGCGTTCTCCCAGGTACGT GAAAGCGCAGCCAGGCGGCTGCTCGECGTTCTCTCAGGCAACTGCTCGGCGTCTCCCAGGTACGT

d:

Gorilla-CR2528 GAAAGCGCAGOCAGGCGGCTGCTCGGCGTTCTCTCAGGTGGCTGCTCGGCGTCTCCCAGGCGGCTGCTCGGCGTTTCCCAGGTACGT GAAAGCGCAGCCAGGCGGCT GCTCGGCGTTCTCTCAGGTGGCTGCTCGGCGTTCTCCCAGG------------------------TACGT

Gorilla-CR2537 GAAAGOGCAGCCAGGCGGCTGCTCGGCGTTCTCTCAGGTGGCTGCTCGGCGITCTCCCAGGCGGCTGCTCGGCGTTTCCCAGGTACGT GAAAGCOCAGCCAGGCGGCTGCTCGGCGTTCTCTCAGGTGGCTGCTCGECGTTCTCCCAGG----------------------TACGT

Gorilla-KSG1471 GAAAGOGCAGOCAGGCGGCTGCTCGGCGTTCTCTCAGGTGGCTGCTCGGCGITCTCCCAGGCGGCTGCTCGGCGITTCCCAGGTACGT

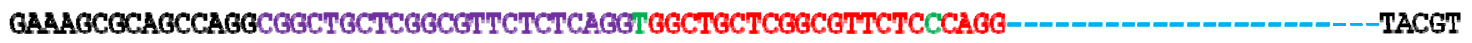

Gorilla-KSG1507 GAAAGOGCAGOCAGGCGGCTGCTCGGCGTTCTCTCAGGTGGCTGCTCGGCGTTCTCCCAGGCGGCTGCTCGGCGTTCTCCCAGGTACGT GAAAGCOCABCCAGGCGGCTGCTCGGCGTTCTCTCAGGTGGCTGCTCGGCGTTCTCCCAGG-----------------------TACGT

Gorilla-KSG2140 GAAAGCGCAGOCAGGCGGCTGCTCGGCGTTCTCTCAGGTGGCTGCTCGGCGITCTCCCAGGCGGCTGCTCGGCGITCTCCCAGGTACGT

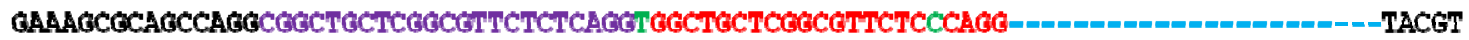

Gorilla-KSG2270 GAAAGCGCAGOCAGGCGGCTGCTCGGCGTTCTCTCAGGTGGCTGCTCGGCGTCTCCCAGGCGGCTGCTCGGCGTTCTCCCAGGTACGT GAAAGCGCAGCCAGGCGGCTGCTCGGCGTTCTCTCAGGTGGCTGCTCGGCGTTCTCCCAGG----------------------TACGT

GOrilla-GTCO2 GAAGCBCAGOCAGGCGGCTGCTCGGCGTTCTCTCAGGTGGCTGCTCGGCGHCTCCCAGGCGGCTGCTCGGCGHCTCCCAGGTACGT GAAAGCGCAGCCAGGCGGCTGCTCGGCOTTCTCTCAGGT GGCTGCTCGGCGTTCTCCCAGG-----------------------TACGT Gor111a-6T007 GAAGGCBCAGOCAGGCGGCTCCTCGGCGTTCTCTCAGGTCOCTOCTCGOCOHTTCCCAGGTACGT GAAAGCOCAGCCAGGCGGCTGCTCGOCGITCTCTCAGGTGGCTGCTCBGCGTTCTCCCAGGTACOT

Figure-1. Multiple sequence alignment of 1st exon flanking TLR2 (23bp ins/del) polymorphism site in primates. (a) 5' UTR sequences in Human, Chimpanzee, marmoset, rhesus monkey, baboon were obtained from NCBI data base and Gorilla sequence from Ensembl genome browser. Neanderthal sequence was obtained from whole genome sequence database. The alignment was performed by webbased multiple sequence alignment programmer (CLUSTAL 2.0.12). Neanderthal (VI 33.25, VI 33.26) 
and gorilla sequences were aligned manually. Results revealed that Marmoset, Rhesus monkey and Baboon harbored del allele, and chimpanzee, orangutan and Neanderthals displayed ins allele. Gorilla TLR2 5'UTR displayed a double insertion sequence (b) To validate the above sequence in database, TLR2 5'UTR region was amplified using genomic DNA of 4 Rhesus monkeys, 3 Baboons, 25 Chimpanzees and 8 Gorilla samples. The amplicons were sequenced and aligned by web based multiple sequence alignment programmer. Lower primates Rhesus monkeys and Baboon displayed homozygous deletion allele, while higher primates viz. chimpanzee harbored insertion of $23 \mathrm{bp}$ nucleotides in both the strands. Out of eight gorilla sequenced for TLR2 Indel polymorphism, seven were heterozygous for double insertion type and one was homozygous for single insertion similar to twenty five chimpanzees investigated in this study.

\section{Figure-2}

a)

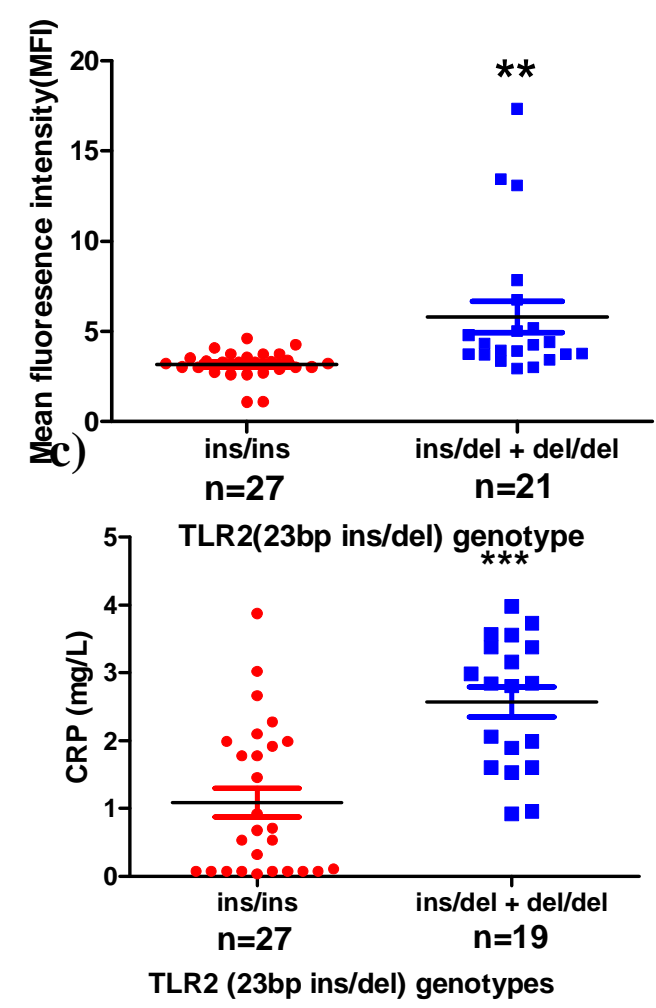

b)
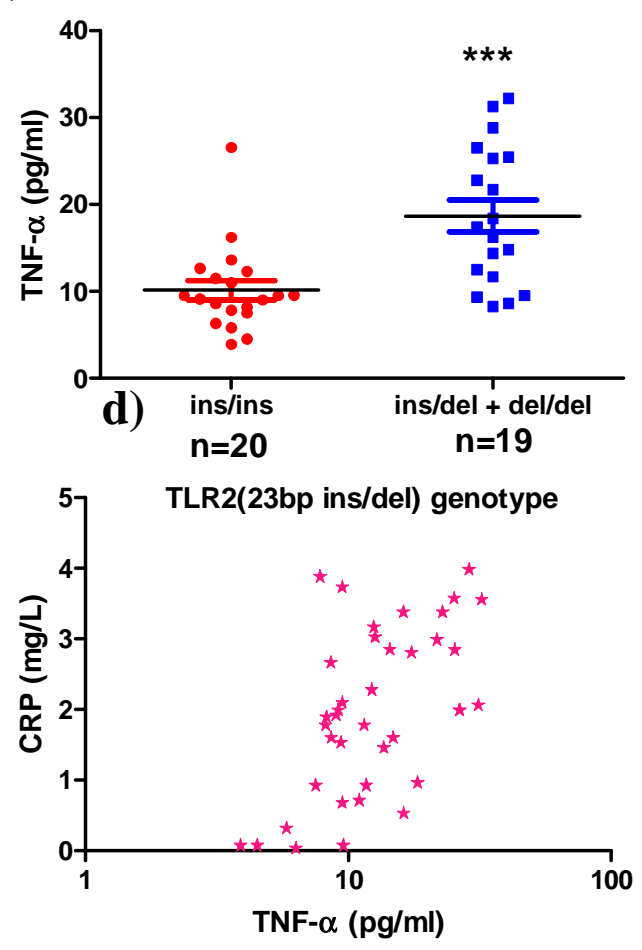
Figure-2. TLR2 (23bp ins/del) genotype is associated with higher TLR2 expression and plasma markers of inflammation: PBMCs of healthy laboratory volunteers were stained for TLR2 and CD14+ monocytes were analyzed by flow cytometry; TNF- $\alpha$ and CRP were quantified in their plasma. a: Mean fluorescence intensity (MFI) of surface TLR2 expression after normalization with isotype control. TLR2 expression was significantly more $(\mathrm{P}<0.0001)$ in subjects with 'del' allele in comparison to those with 'ins' allele. Plasma levels of TNF- $\alpha$ (b) and CRP (c) were quantified by ELISA and a turbidolatex method respectively. Significantly higher levels of both plasma TNF- $\alpha(\mathrm{P}<0.001)$ and CRP (unpaired $t$ test $\mathrm{P}<0.001$ ) was observed in 'del' carriers; (d) A significant positive correlation was demonstrable between plasma CRP and TNF- $\alpha$ levels in 46 healthy controls $(\mathrm{P}<0.0001$, Spearman $r=0.53)$. Significance in figure 1a remained even when the three out layers were not factored-in for statistical analysis.

\section{Figure-3}

a)

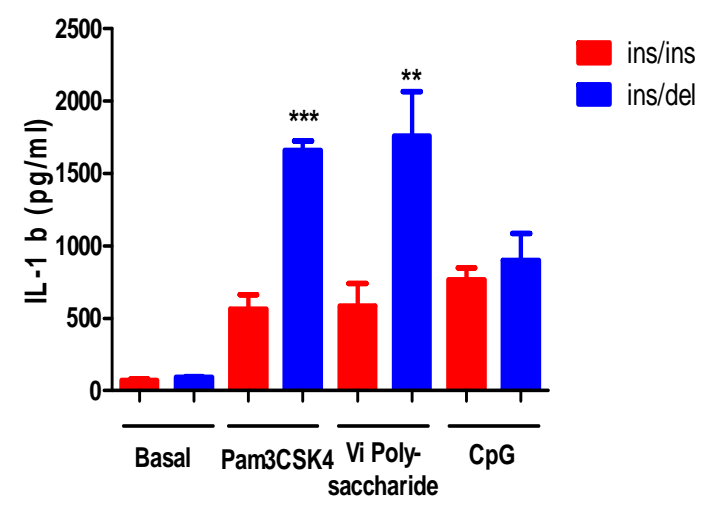

c)

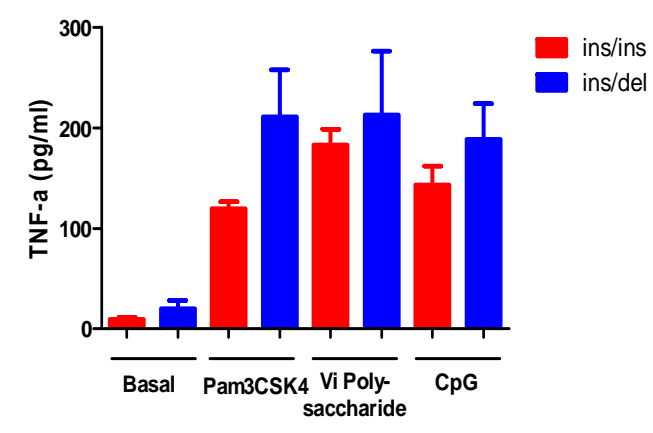

b)

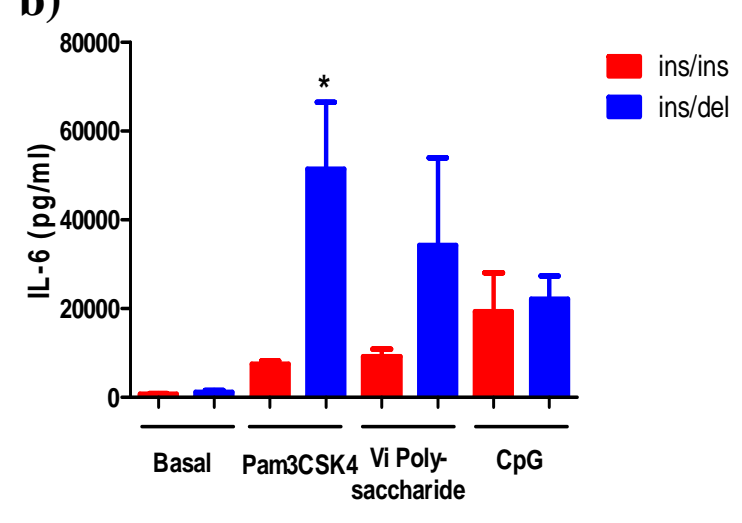

d)

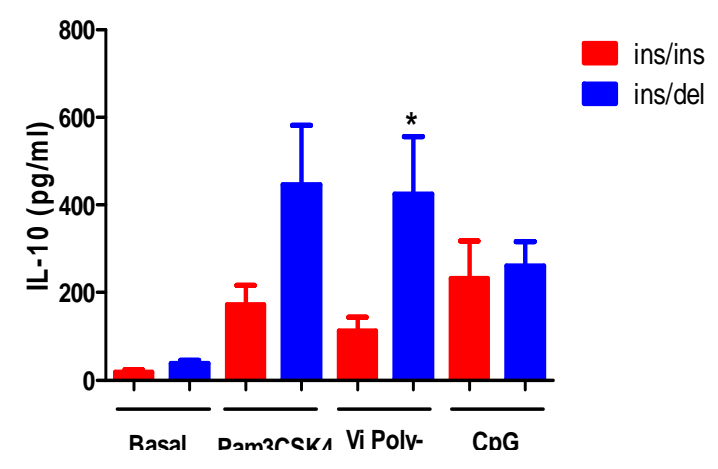


e)

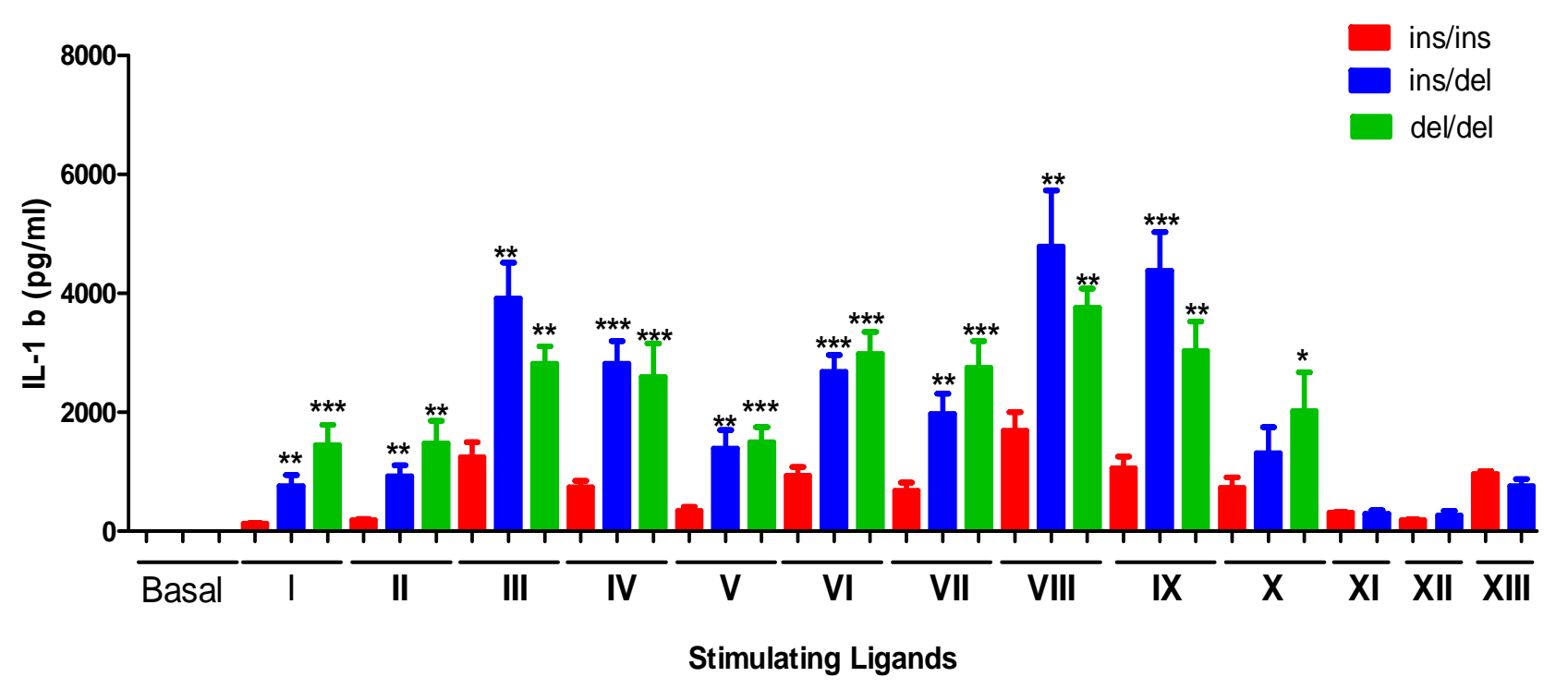

Figure-3. TLR2 ligands induce enhanced levels of cytokines in PMBCs of 'del' allele carriers: Healthy controls were screened for TLR2 (23bp ins/del), TLR4 (Asp299Gly and Thr399Ile) and TLR1 (I602S) polymorphisms as described in methods section. Individuals, with wildtype TLR4 and TLR1 polymorphisms i.e. Asp299Asp, Thr399Thr and I602I but displaying 'del' or 'ins' of TLR2 were tested. Normal human PBMCs with ins/ins $(n=5)$ or ins/del $(n=5)$ were stimulated in separate matrixes with Pam3CSK4 (1ng/ml), Vi polysaccharide (20ug/ml), CpG (10ug/ml) or LPS (10ug/ml) for 48hrs. Polymixin B (10ug/ml) was used in culture medium except in positive controls where LPS was employed as a ligand. In all assays LPS with polymixin-B was taken as negative control to ensure non-induction of IL1 $\beta$. Culture supernatants were quantified for (a) IL-1b, (b) IL-6, (c) TNF- $\alpha$ and (d) IL-10 by Bio-plex bead array (Biorad). Upon stimulation with Pam3CSK44, cells of ins/del subjects produced significantly higher IL-1b $(\mathrm{P}<0.001)$ and IL-6 $(\mathrm{P}<0.05)$ when compared to ins/ins subjects; Vi polysaccharide of S.typhi induced higher IL-1b $(\mathrm{P}<0.01)$ and IL-10 $(\mathrm{P}<0.05)$ in ins/del carriers. CpG DNA a TLR-9 ligand induced comparable levels of four cytokines in both the groups of subjects; (e), human PBMCs from 8 ins/ins, 8 ins/del 5 and del/del subjects were stimulated with (I) Pam3CSK44 (1ng/ml), (II) Vi polysaccharide (20ug/ml), (III) Peptidoglycan (PGN) from Streptomyces (100ug/ml), (IV) PGN from Methano species (100ug/ml), (V) PGN from Saccharomyces cereviseae (100ug/ml), (VI) PGN from Micrococcus luteus (100ug/ml), (VII) PGN from Bacillus subtilis (100ug/ml), (VIII) PGN from 
Staphylococcus aureus (100ug/ml) and (IX) $\gamma$ - irradiated Mycobacterium tuberculosis $\left(1 \mathrm{X} 10^{6} / \mathrm{ml}\right)$. (X): LPS (10ug/ml) was taken as positive control. As controls PBMCs from 6 ins/ins and 6 ins/del carriers were stimulated with (XI) CpG (10ug/ml), (XII) Poly IC (10ug/ml) and (XIII) Flagellin (100ug/ml). IL$1 \mathrm{~b}$ levels were measured in all culture supernatants by ELISA and analyzed by student's t test. P value less than 0.05 was considered as significant $(*=\mathrm{P}<0.05$, $* *=\mathrm{P}<0.01$ and $* * *=\mathrm{P}<0.001)$. Subjects with 'del' allele consistently secreted significantly higher levels of IL-1b when stimulated with any of the TLR2 ligands while supernatants of cultures stimulated with $\mathrm{CpG}$, Poly IC and Flagellin displayed comparable levels of IL-1b. Polymyxin-B at a concentration of $10 \mathrm{ug} / \mathrm{ml}$ completely inhibited LPS mediated activation (which was taken in each plate) - IL-1b levels below 10pg/ml in LPS stimulated cavities (with Polymyxin-B) were treated as invalid experiments.

\section{Figure-4}

a)

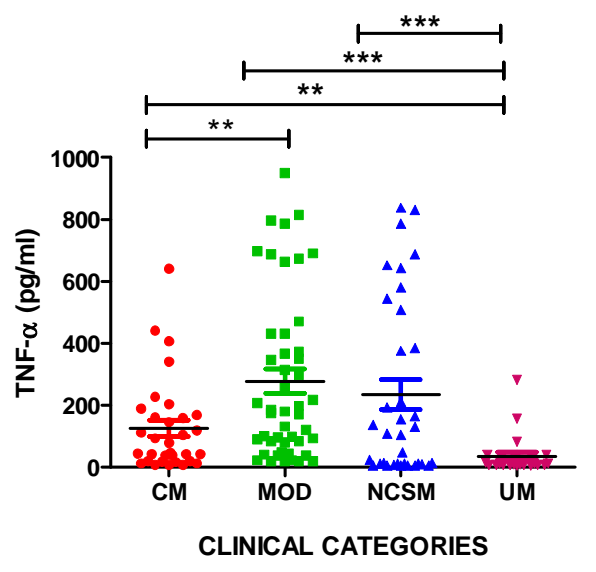

c)

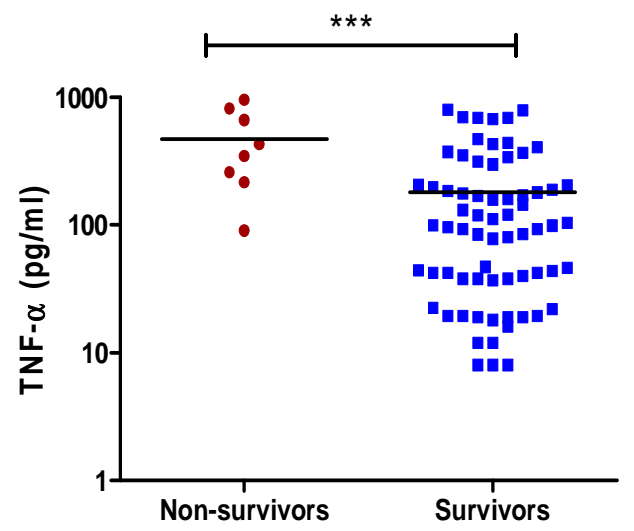

b)

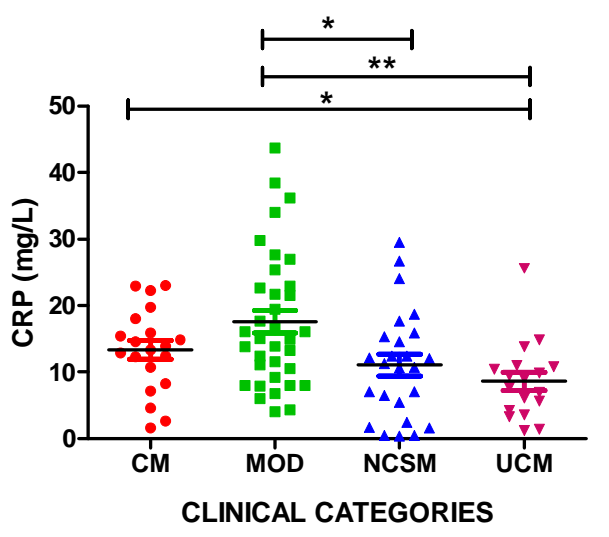

d)

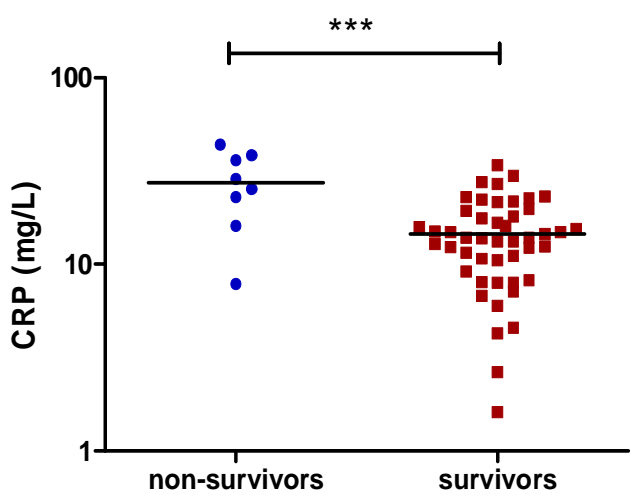


Figure-4. Plasma TNF- $\alpha$ and CRP levels correlate with clinical severity and mortality in malaria. (a,b): Plasma TNF- $\alpha$ and CRP levels were determined by ELISA (CM, n=32; MOD, n=47; NCSM, n=35 and UM, n=24) and turbidometric assays (CM, n=20; MOD, n=35; NCSM, n=25 and UM, n=18), respectively in samples collected from patients before treatment with anti-malarials. CM and MOD cases were enrolled for correlation between TNF- $\alpha$ and CRP levels with mortality. Plasma levels of TNF- $\alpha$ (c) and CRP (d) was significantly more in patients who died when compared with survivors. (*P<0.05, $* * \mathrm{P}<0.01, * * * \mathrm{P}<0.001)$

\section{Figure-5}

a)

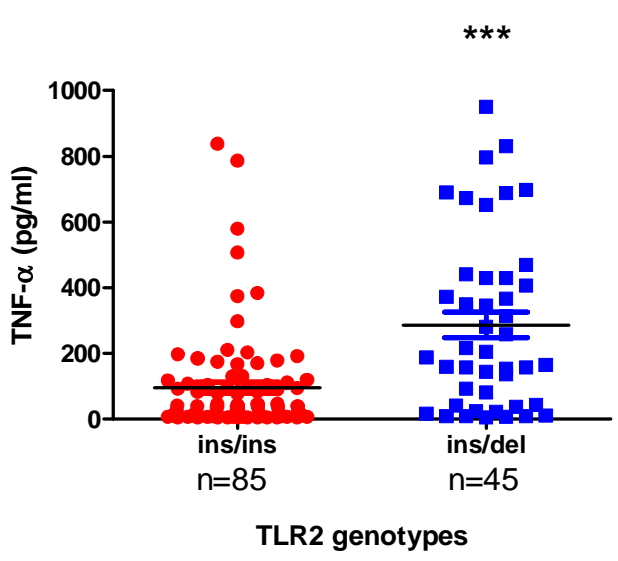

c)

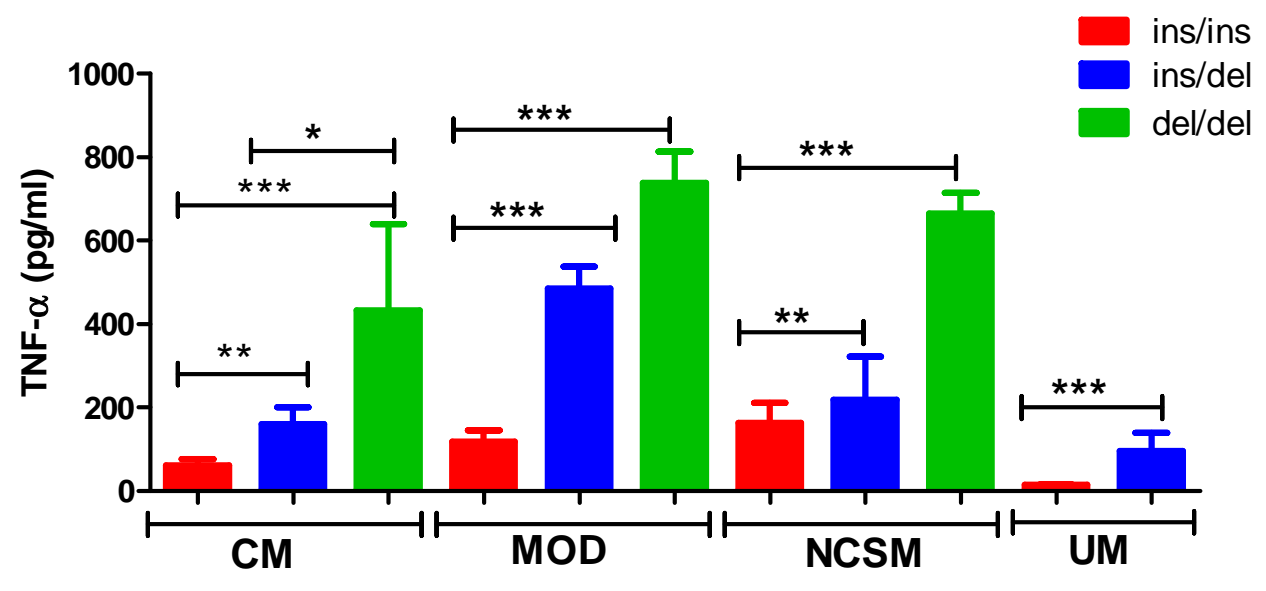


d)

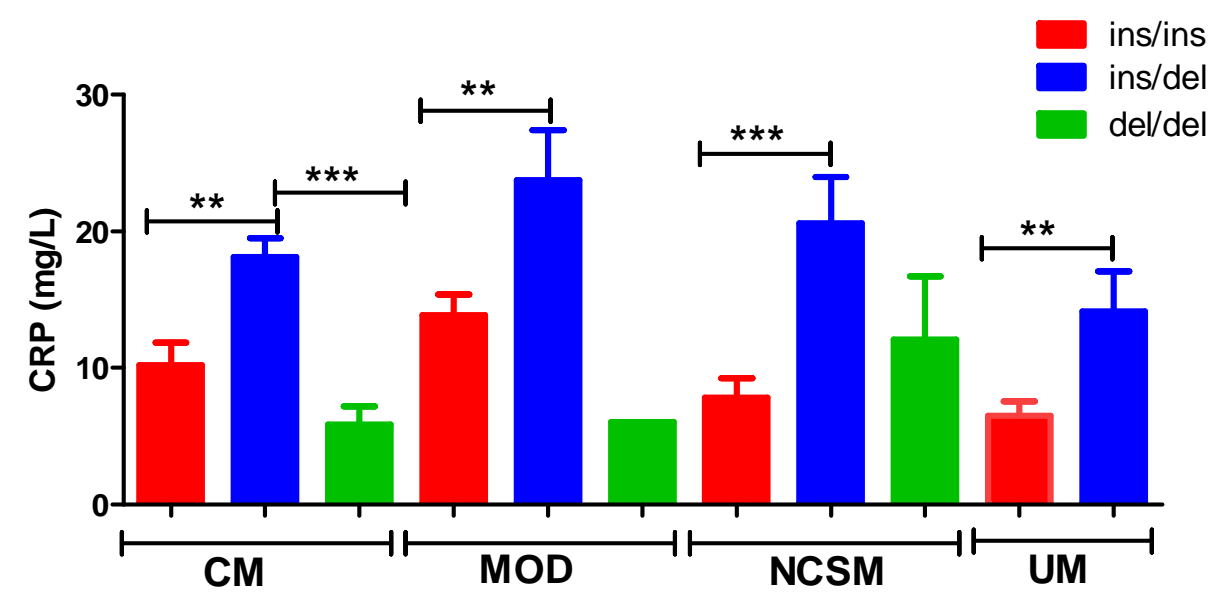

Figure-5 TLR2 'del' allele is associated with higher plasma levels of TNF- $\alpha$ and CRP in $P$.

falciparum infected patients. Plasma concentrations of (a) TNF- $\alpha$ and (b) CRP were measured by ELISA and Turbidolatex, respectively in plasma of $P$. falciparum infected patients (combined clinical categories) and correlated with 'del' or 'ins' genotype of TLR2 gene. Scatter plots show data for TNF- $\alpha$ in 130 patients and for CRP in 87 patients. Mean plasma levels of TNF- $\alpha$ and CRP were significantly elevated in malaria patients with ins/del genotype in comparison to patients with ins/ins (unpaired t test, $* * * \mathrm{P}<0.001$ ).(c) Plasma TNF- $\alpha$ levels in clinical categories of $P$. falciparum malaria viz. CM, cerebral malaria (ins/ins=17; ins/del=13; del/del=2); MOD, multi organ dysfunction (ins/ins=28; ins/del=17; del/del=2); NCSM, non-cerebral severe malaria (ins/ins=22; ins/del=9; del/del=4); UM, uncomplicated malaria (ins/ins=18; ins/del=6) were analysed by student's $\mathrm{t}$ test in various genotypes. (*P<0.05, **P<0.01, ***P<0.001) (d) Plasma levels of CRP was compared different TLR2 indel genotypes in various clinical categories of $P$. falciparum malaria $(* * \mathrm{P}<0.01, * * * \mathrm{P}<0.001)$.

\section{Figure-6}

a)

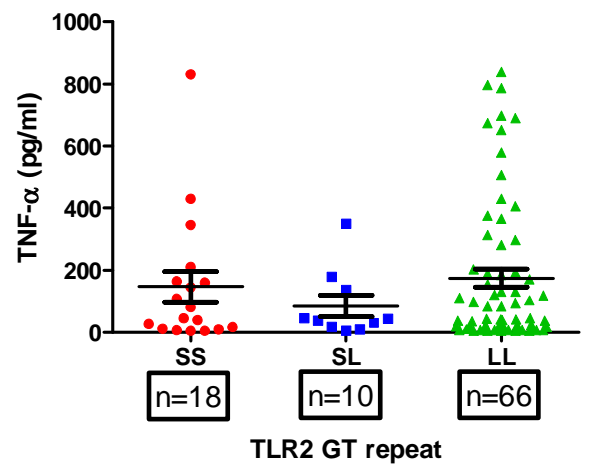

b)

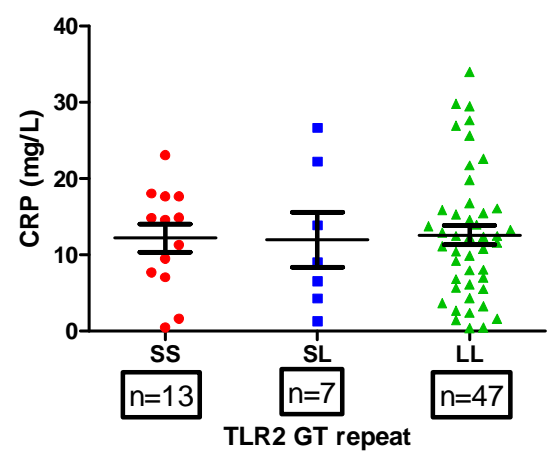




$\begin{array}{llllll}\text { Studied area } & \text { Number of healthy } & \text { Mean age } & \text { ins allele } & \text { del allele } & \text { Reference } \\ \text { subjects } & \text { (years) } & (\%) & (\%) & \end{array}$

c)

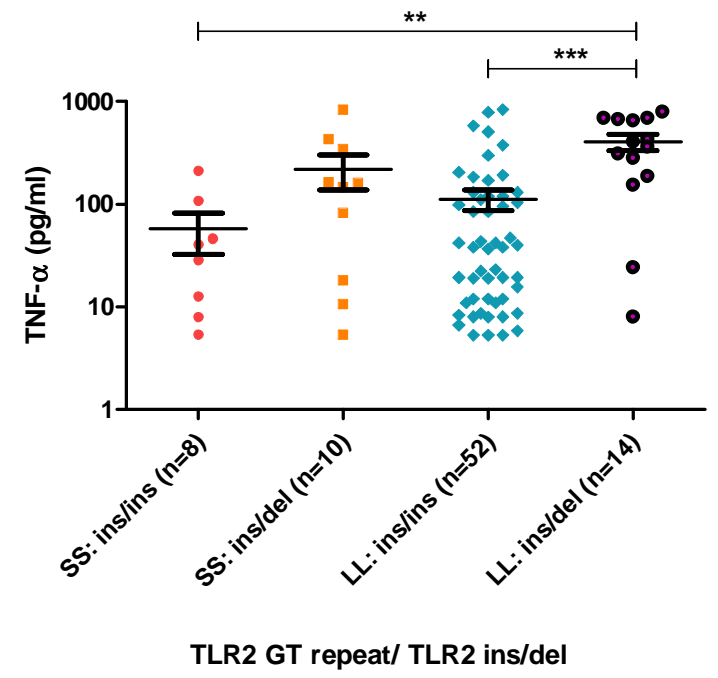

d)

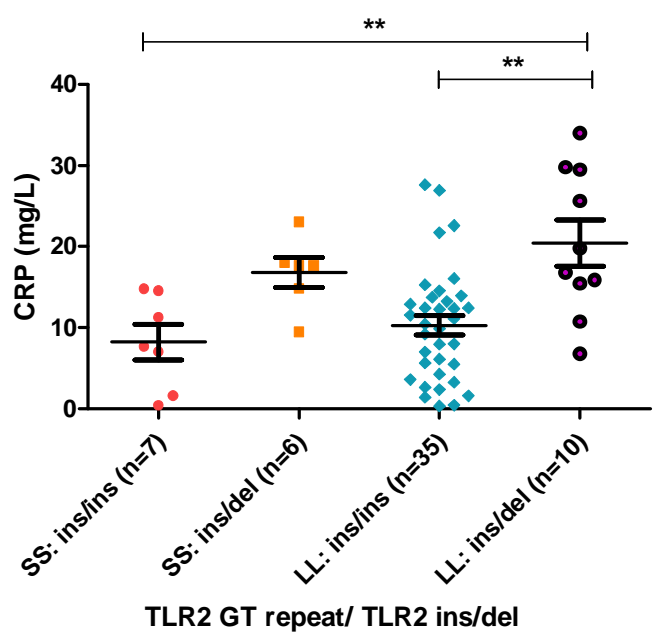

Figure- 6 Plasma TNF- $\alpha$ and CRP levels are not correlated TLR2 (GT repeat) in P. falciparum malaria patients. TLR2 GT repeat was typed by PCR-fragment analysis. Alleles were classified in to two broad group $\mathrm{S}$ ( $<11$ repeat) and $\mathrm{L}$ (16-21 repeats). Plasma concentrations of TNF- $\alpha$ and CRP were measured by ELISA and Turbidolatex, respectively in plasma of $P$. falciparum infected patients (combined clinical categories) and correlated with GT repeat alleles (S and L) of TLR2 gene. Mean plasma levels of TNF- $\alpha$ and CRP were comparable (a and b). On combined analysis of TLR2 (23bp ins/del) and TLR2 GT repeat polymorphism (c and d), patients with LL : ins/del genotypes combination displayed higher plasma TNF- $\alpha$ and CRP when compared to SS: ins/ins and LL: ins/ins combinations. $(* \mathrm{P}<0.05, * * \mathrm{P}<0.01$, $* * * \mathrm{P}<0.001)$ 


\begin{tabular}{|c|c|c|c|c|c|}
\hline PNG & 333 & NA* & 87 & 13 & ${ }^{31}$ Greene et al. 2009 \\
\hline Kenya & 153 & $\mathrm{NA}^{*}$ & 78.5 & 31.5 & ${ }^{31}$ Greene et al. 2009 \\
\hline $\begin{array}{l}\text { North America } \\
\text { (African) }\end{array}$ & 93 & NA* & 79 & 21 & ${ }^{31}$ Greene et al. 2009 \\
\hline $\begin{array}{l}\text { North America } \\
\text { (Caucasian) }\end{array}$ & 84 & NA* & 87 & 13 & ${ }^{31}$ Greene et al. 2009 \\
\hline $\begin{array}{l}\text { North America } \\
\text { (Asian) }\end{array}$ & 84 & NA* & 75 & 25 & ${ }^{31}$ Greene et al. 2009 \\
\hline Northern Han & 400 & 81.4 & 64 & 36 & ${ }^{32}$ Yu et al. 2011 \\
\hline China & & & & & \\
\hline Brazil & 225 & 56.5 & 92 & 8 & ${ }^{33}$ Oliveira et al. 2012 \\
\hline North India & 250 & 64.7 & 88 & 12 & ${ }^{34}$ Mandal et al. 2012 \\
\hline Germany & 347 & 58.5 & 85 & 15 & ${ }^{35}$ Nischalke et al. 2011 \\
\hline Japan & 146 & 36 & 72 & 28 & ${ }^{22}$ Tahara et al. 2007 \\
\hline Greece & 480 & 60.77 & 95 & 5 & $\begin{array}{l}{ }^{38} \text { Theodoropoulos et al. } \\
2012\end{array}$ \\
\hline France & 196 & NA & 85 & 15 & ${ }^{39}$ Oliveira et al. 2014 \\
\hline Poland & 304 & NA & 82 & 18 & $\begin{array}{l}{ }^{40} \text { Lewandowska et al. } \\
2015\end{array}$ \\
\hline Tunisia & 260 & 53 & 82.5 & 17.5 & ${ }^{41}$ Zidi et al. 2016 \\
\hline Egypt & 100 & NA & 77.5 & 22.5 & ${ }^{42} \mathrm{Al}-$ Harras et al. 2016 \\
\hline North India & 270 & NA & 66 & 34 & ${ }^{35}$ Vidyant et al. 2017 \\
\hline North Iran & 50 & 47.7 & 81 & 19 & ${ }^{43}$ Habibzadeh et al. 2017 \\
\hline Eastern India & 432 & 32 & 86 & 14 & Present study \\
\hline
\end{tabular}

Table-1 Distribution of TLR2 23bp indel polymorphism in various populations 
Table-2 Characteristic of $\boldsymbol{P}$. falciparum infected Indian patients at hospital admission.

\begin{tabular}{|c|c|c|c|c|}
\hline Characteristics & $\mathrm{CM}(\mathrm{n}=97)$ & MOD $(n=123)$ & $\operatorname{NCSM}(n=75)$ & UCM $(n=103)$ \\
\hline $\operatorname{Sex}(M / F)$ & $73 / 24$ & $97 / 26$ & $60 / 15$ & $79 / 24$ \\
\hline Mean age in years (range) & $30.77(15-86)$ & $32.09(15-83)$ & $33.02(15-70)$ & $31.19(15-75)$ \\
\hline $\begin{array}{l}\text { Hemoglobin g/dl, mean } \pm \\
\text { SD }\end{array}$ & $9.879 \pm 2.028$ & $9.848 \pm 2.206$ & $9.704 \pm 2.488$ & $10.54 \pm 1.867$ \\
\hline GCS (mean (range) & $8.598(3-11)$ & $7.98(3-15)^{*}$ & -- & -- \\
\hline Neutrophils \%,mean(range) & $68.52(42-92)$ & $70.2(37-92)$ & $67.64(31-88)$ & $65.88(33-92)$ \\
\hline Lymphocytes \%,mean(range) & $27.15(10-54)$ & $26.33(5-59)$ & $27.10(0-65)$ & $29.32(7-55)$ \\
\hline Monocytes \%, mean (range) & $1.57(0-4)$ & $1.58(0-4)$ & $1.38(0-5)$ & $1.21(0-6)$ \\
\hline Eosinophils \%, mean (range) & $3.11(0-12)$ & $2.82(1-7)$ & $3.19(2-16)$ & $3.17(0-10)$ \\
\hline $\begin{array}{l}\text { Total leukocyte count, } \\
\text { mean } \pm \text { SD }\end{array}$ & $8431 \pm 2097$ & $9299 \pm 2875$ & $8512 \pm 2433$ & $7850 \pm 2162$ \\
\hline $\mathrm{TPC}$ mean $\pm \mathrm{SD}$ & $188333 \pm 52915$ & $178625 \pm 67712$ & $174720 \pm 74581$ & $197267 \pm 64898$ \\
\hline $\begin{array}{l}\text { Fasting blood sugar mg/dl, } \\
\text { mean } \pm \text { SD }\end{array}$ & $148 \pm 84.21$ & $141.7 \pm 98.34$ & $138.5 \pm 82.40$ & $120.7 \pm 56.0$ \\
\hline SGOT units/ml, m \pm SD & $30.13 \pm 7.99$ & $169.9 \pm 120.7 * *$ & $130.8 \pm 78.19 * *$ & $33.96 \pm 8.48$ \\
\hline SGPT units/ml, $\mathrm{m} \pm \mathrm{SD}$ & $27.47 \pm 8.94$ & $130.5 \pm 75.48 * *$ & $109.5 \pm 54.28 * *$ & $28.08 \pm 9.97$ \\
\hline$A L P$, mean \pm SD & $170.8 \pm 93.16$ & $221.7 \pm 156.6$ & $190 \pm 141.7$ & $158.3 \pm 82.2$ \\
\hline Serum urea $\mathrm{mg} / \mathrm{dl}, \mathrm{m} \pm \mathrm{SD}$ & $53.31 \pm 28.82$ & $142.5 \pm 71.85^{* * * *}$ & $169.3 \pm 73.00 * * *$ & $37.73 \pm 19.04$ \\
\hline $\begin{array}{l}\text { Serum creatinine } \mathrm{mg} / \mathrm{dl} \text {, } \\
\text { mean } \pm \mathrm{SD}\end{array}$ & $1.36 \pm 0.63$ & $5.03 \pm 3.38 * * *$ & $6.13 \pm 3.05 * * *$ & $1.15 \pm 0.34$ \\
\hline $\mathrm{Na}^{+} \mathrm{mEq} / \mathrm{L}$, mean $\pm \mathrm{SD}$ & $136.2 \pm 8.07$ & $134.4 \pm 9.51$ & $133.3 \pm 6.47$ & $135.9 \pm 7.96$ \\
\hline $\mathrm{K}^{+} \mathbf{m E q} / \mathbf{L}$, mean $\pm \mathrm{SD}$ & $4.3 \pm 0.91$ & $4.51 \pm 1.12$ & $4.21 \pm 0.8$ & $4.17 \pm 0.73$ \\
\hline $\mathrm{Mg}^{++} \mathrm{mEq} / \mathrm{L}$, mean $\pm \mathrm{SD}$ & $3.48 \pm 2.06$ & $3.27 \pm 1.34$ & $3.34 \pm 1.58$ & $3.52 \pm 3.30$ \\
\hline $\mathrm{Ca}^{++} \mathrm{mEq} / \mathrm{L}$, mean $\pm \mathrm{SD}$ & $0.87 \pm 0.20$ & $0.99 \pm 0.15$ & $0.89 \pm 0.19$ & $0.92 \pm 0.21$ \\
\hline Case fatality rate, no (\%) & $13(14)$ & $36(32)$ & $\mathbf{0}$ & $\mathbf{0}$ \\
\hline
\end{tabular}


Table 3. Prevalence of Genotype and allele of TLR2 (23bp ins/del) polymorphism in P.falciparum malaria patients.

\begin{tabular}{|c|c|c|c|c|c|c|c|c|c|c|c|c|}
\hline & \multicolumn{4}{|c|}{$\begin{array}{l}\text { Clinical categories, } \\
(\%) \text { of cases }\end{array}$} & \multicolumn{2}{|l|}{ CM Vs UM } & \multicolumn{2}{|l|}{ MOD Vs UM } & \multicolumn{2}{|c|}{ NCSM Vs UM } & \multicolumn{2}{|c|}{$\begin{array}{l}\text { CM+MOD+NCSM Vs } \\
\text { UM }\end{array}$} \\
\hline & $\begin{array}{l}\text { CM } \\
(n=81)\end{array}$ & $\begin{array}{l}\text { MOD } \\
(n=113)\end{array}$ & $\begin{array}{l}\text { NCSM } \\
(n=67)\end{array}$ & $\begin{array}{l}\text { UM } \\
(n=95)\end{array}$ & $\begin{array}{l}\text { OR }(95 \% \\
\text { CI })\end{array}$ & $\begin{array}{l}\mathbf{P} \\
\text { Value }\end{array}$ & $\begin{array}{l}\text { OR }(95 \% \\
\text { CI) }\end{array}$ & P Value & $\begin{array}{l}\text { OR }(95 \% \\
\text { CI) }\end{array}$ & P value & OR $(95 \% \mathrm{CI})$ & P value \\
\hline ins/ins & $49(60)$ & $69(61)$ & $43(64)$ & $67(71)$ & 1 (ref) & - & 1 (ref) & - & 1 (ref) & - & 1 (ref) & - \\
\hline ins/del & $28(35)$ & $30(27)$ & $20(30)$ & $27(28)$ & $\begin{array}{l}0.70(0.37 \text { to } \\
1.34)\end{array}$ & 0.32 & $\begin{array}{l}0.926(0.49 \\
\text { to } 1.72)\end{array}$ & 0.87 & $\begin{array}{l}1.15(0.57 \\
\text { to } 2.31)\end{array}$ & 0.72 & $\begin{array}{l}0.81(0.47 \text { to } \\
1.41)\end{array}$ & 0.49 \\
\hline del/del & $4(5)$ & $14(12)$ & $4(6)$ & $1(1)$ & $\begin{array}{l}0.18(0.01 \text { to } \\
1.68)\end{array}$ & 0.16 & $\begin{array}{l}13.59(1.73 \\
\text { to } 106.3)\end{array}$ & 0.001 & $\begin{array}{l}0.16(0.01 \\
\text { to } 1.48)\end{array}$ & 0.15 & $\begin{array}{l}10.22(1.33 \text { to } \\
78.32)\end{array}$ & 0.004 \\
\hline $\begin{array}{l}\text { Ins/del } \\
+ \\
\text { del/del }\end{array}$ & $32(40)$ & $44(39)$ & $24(36)$ & $28(29)$ & $\begin{array}{l}0.64(0.34 \text { to } \\
1.19)\end{array}$ & 0.20 & $\begin{array}{l}1.52(0.85 \text { to } \\
2.72)\end{array}$ & 0.18 & $\begin{array}{l}0.74(0.38 \\
\text { to } 1.45)\end{array}$ & 0.39 & $\begin{array}{l}0.64(0.38 \text { to } \\
1.09)\end{array}$ & 0.11 \\
\hline Allele & $2 n=162$ & $2 n=226$ & & $2 n=190$ & & & & & & & & \\
\hline ins & $126(78)$ & $168(74)$ & $106(79)$ & $161(85)$ & 1 (ref) & - & 1 (ref) & - & 1 (ref) & - & 1 (ref) & - \\
\hline del & $36(22)$ & $58(26)$ & $28(21)$ & $29(15)$ & $\begin{array}{l}1.58(0.92 \text { to } \\
2.727)\end{array}$ & 0.09 & $\begin{array}{l}1.91(1.16 \text { to } \\
3.14)\end{array}$ & 0.01 & $\begin{array}{l}1.46(0.82 \\
\text { to } 2.60)\end{array}$ & 0.23 & $\begin{array}{l}1.77(1.12 \text { to } \\
2.80)\end{array}$ & 0.01 \\
\hline
\end{tabular}


Table-4. Association of TLR2 (23bp ins/del) polymorphism with treatment out come in severe $P$. falciparum malaria.

\begin{tabular}{lllll}
\hline Genotype/Allele & Death & Survivor & P value & OR $(95 \%$ CI $)$ \\
\hline Genotype & $(\mathrm{n}=36)$ & $(\mathrm{n}=158)$ & & \\
ins/ins & $16(44)$ & $102(65)$ & - & $1($ ref $)$ \\
ins/del & $13(36)$ & $45(28)$ & 0.19 & $1.84(0.81$ to 4.14$)$ \\
del/del & $7(20)$ & $11(7)$ & 0.01 & $4.05(1.37$ to 12.00) \\
ins/del + del/del & $20(56)$ & $56(35)$ & 0.03 & $2.27(1.09$ to 4.74$)$ \\
Allele & $(2 n=72)$ & $(2 n=316)$ & & \\
ins & $45(63)$ & $249(79)$ & & \\
del & $27(37)$ & $67(21)$ & 0.005 & $2.23(1.28$ to 3.85) \\
\hline
\end{tabular}

NOTE: Data are no. (\%) of participants unless otherwise specified;

OR: odds ratio; $\mathrm{CI}$ : confidence interval 